\title{
Analysis of Saturn's thermal emission at 2.2-cm wavelength: Spatial distribution of ammonia vapor
}

\author{
A.L. Laraia ${ }^{\text {a,* }}$, A.P. Ingersoll ${ }^{\text {a }}$, M.A. Janssen ${ }^{\text {b }}$, S. Gulkis ${ }^{\text {b }}$, F. Oyafuso ${ }^{\text {b }}$, M. Allison ${ }^{c}$ \\ ${ }^{a}$ California Institute of Technology, Pasadena, CA 91125, United States \\ ' Jet Propulsion Laboratory, California Institute of Technology, Pasadena, CA 91109, United States \\ ${ }^{c}$ NASA Goddard Institute for Space Studies, New York, NY 10025, United States
}

\section{A R T I C L E I N F O}

\section{Article history:}

Available online 27 June 2013

\section{Keywords:}

Saturn, Atmosphere

Atmospheres, Structure

Atmospheres, Composition

Atmospheres, Dynamics

Radio observations

\begin{abstract}
A B S T R A C T
This work focuses on determining the latitudinal structure of ammonia vapor in Saturn's cloud layer near 1.5 bars using the brightness temperature maps derived from the Cassini RADAR (Elachi et al. [2004], Space Sci. Rev. 115, 71-110) instrument, which works in a passive mode to measure thermal emission from Saturn at $2.2-\mathrm{cm}$ wavelength. We perform an analysis of five brightness temperature maps that span epochs from 2005 to 2011, which are presented in a companion paper by Janssen et al. (Janssen, M.A., Ingersoll, A.P., Allison, M.D., Gulkis, S., Laraia, A.L., Baines, K., Edgington, S., Anderson, Y., Kelleher, K., Oyafuso, F. [2013]. Icarus, this issue). The brightness temperature maps are representative of the spatial distribution of ammonia vapor, since ammonia gas is the only effective opacity source in Saturn's atmosphere at $2.2-\mathrm{cm}$ wavelength. Relatively high brightness temperatures indicate relatively low ammonia relative humidity ( $\mathrm{RH})$, and vice versa. We compare the observed brightness temperatures to brightness temperatures computed using the Juno atmospheric microwave radiative transfer (JAMRT) program which includes both the means to calculate a tropospheric atmosphere model for Saturn and the means to carry out radiative transfer calculations at microwave frequencies. The reference atmosphere to which we compare has a $3 \times$ solar deep mixing ratio of ammonia (we use $1.352 \times 10^{-4}$ for the solar mixing ratio of ammonia vapor relative to $\mathrm{H}_{2}$; see Atreya [2010]. In: Galileo's Medicean Moons - Their Impact on 400 years of Discovery. Cambridge University Press, pp. 130-140 (Chapter 16)) and is fully saturated above its cloud base. The maps are comprised of residual brightness temperaturesobserved brightness temperature minus the model brightness temperature of the saturated atmosphere.

The most prominent feature throughout all five maps is the high brightness temperature of Saturn's subtropical latitudes near $\pm 9^{\circ}$ (planetographic). These latitudes bracket the equator, which has some of the lowest brightness temperatures observed on the planet. The observed high brightness temperatures indicate that the atmosphere is sub-saturated, locally, with respect to fully saturated ammonia in the cloud region. Saturn's northern hemisphere storm was also captured in the March 20, 2011 map, and is very bright, reaching brightness temperatures of $166 \mathrm{~K}$ compared to $148 \mathrm{~K}$ for the saturated atmosphere model. We find that both the subtropical bands and the 2010-2011 northern storm require very low ammonia RH below the ammonia cloud layer, which is located near 1.5 bars in the reference atmosphere, in order to achieve the high brightness temperatures observed. The disturbances in the southern hemisphere between $-42^{\circ}$ and $-47^{\circ}$ also require very low ammonia $\mathrm{RH}$ at levels below the ammonia cloud base. Aside from these local and regional anomalies, we find that Saturn's atmosphere has on average $70 \pm 15 \%$ ammonia relative humidity in the cloud region. We present three options to explain the high $2.2-\mathrm{cm}$ brightness temperatures. One is that the dryness, i.e., the low $\mathrm{RH}$, is due to higher than average atmospheric temperatures with constant ammonia mixing ratios. The second is that the bright subtropical bands represent dry zones created by a meridionally overturning circulation, much like the Hadley circulation on Earth. The last is that the drying in both the southern hemisphere storms and 20102011 northern storm is an intrinsic property of convection in giant planet atmospheres. Some combination of the latter two options is argued as the likely explanation.
\end{abstract}

() 2013 Elsevier Inc. All rights reserved.

\footnotetext{
* Corresponding author.

E-mail address: alaraia@caltech.edu (A.L. Laraia).
}

\section{Introduction}

The instruments on board the Cassini orbiter have provided the giant planets community with a plethora of data on Saturn's 
atmosphere for the past decade. Ideally, we would like to get a comprehensive picture of Saturn's atmosphere that reconciles the general circulation, the cloud and haze distributions and compositions, the zonal wind profile, and the storm locations and dynamics. One major observational roadblock is that the stratospheric and upper tropospheric clouds and hazes on Saturn block our view of the atmosphere beneath them.

The location and magnitude of the zonal jets at the cloud tops are well known from Voyager measurements (Sánchez-Lavega et al., 2000). The broad, strongly superrotating jet centered on the equator is a distinctive feature, with alternating eastward and westward jets to either side of the equator. Unlike Jupiter, convection on Saturn appears in both westward and eastward jets (Del Genio et al., 2009). Convective events on Saturn are intermittent, and the cause of the intermittency is uncertain. Saturn electrostatic discharges, or SEDs (Kaiser et al., 1983; Porco et al., 2005; Fischer et al., 2006, 2007), have been observed in convective storms and are indicative of lightning at depth. What causes these convective outbursts on Saturn, and how do they contribute to or maintain the general circulation? How does deep convection work on Saturn, and how does it fit together with the latitudinal belt-zone structure of the giant planets? Answers to these questions have been difficult to obtain. The $2.2-\mathrm{cm}$ observations analyzed in this work provide new data on the distribution of ammonia vapor in and beneath the ammonia clouds, and will help diagnose the atmospheric dynamics at work inside the convective storms.

The structure of Saturn's clouds and hazes is still being studied, although the general features are understood. The equatorial zone is a region of constant high clouds and thick haze, whereas the midlatitudes (generally between $\pm 20^{\circ}$ and $\pm 60^{\circ}$ ) are regions of smaller, more variable clouds (West et al., 2009). The vertical structure and composition of these clouds and hazes is not well known, but Cassini observations made by the ISS (imaging science subsystem), VIMS (Visual and Infrared Mapping Spectrometer) and CIRS (Composite Infrared Spectrometer) instruments are closing our knowledge gaps in these areas. Tied to the distribution of clouds and hazes is the distribution of tropospheric gases, for example ammonia and phosphine. How does the latitudinal distribution of clouds, hazes, and tropospheric gases coincide with Saturn's belt-zone structure? Knowing the spatial distribution of these gases can help us determine the dynamical mechanisms that produce the spatial patterns themselves. For example, vertical motion, caused by either convection or large-scale meridional overturning, plays a key role in determining where clouds and hazes will or will not form.

This work focuses on determining the latitudinal structure of ammonia vapor in Saturn's ammonia cloud layer using the brightness temperature maps derived from the Cassini RADAR (Elachi et al., 2004) instrument, which works in a passive mode to measure thermal emission from Saturn at 2.2-cm wavelength. These maps are presented in a companion paper by Janssen et al. (2013, this issue), hereafter referred to as J13. The maps provide data on the spatial distribution of ammonia vapor in the pressure range 1-2 bars, in the vicinity of the ammonia ice cloud. We believe these maps provide information about Saturn's meridional circulation. The 2.2-cm data have better spatial resolution and sensitivity than any other microwave data on Saturn. The calibration of Cassini's RADAR instrument, described in detail in Janssen et al. (2009) and $\mathrm{J} 13$, is accurate and was validated using both Saturn and more recent Titan observations as described in J13.

Section 2 describes the $2.2-\mathrm{cm}$ observations and the radiative transfer model used in our analysis. The brightness temperature maps are described in Section 3. Section 4 compares the observations to the output from the radiative transfer model. Discussion and implications for Saturn's atmospheric dynamics are given in Section 5, and conclusions are given in Section 6.

\section{Observations and radiative transfer model}

Cassini's RADAR radiometer was used to map Saturn during five equatorial periapsis passes occurring between 2005 and 2011. The maps were formed from continuous pole-to-pole scans taken through Saturn nadir during the periapsis passes, allowing the rotation of Saturn to sweep the scan westward in longitude. The observations and mapping are described in detail in J13 along with the calibration and error analysis. We refer the reader to Section 2 of J13 for a description of the observations and observational approach, and to Section 3.2 of J13 for a description of the map-generating process.

The reference model used to calculate the residual brightness temperature maps is also described in detail in Section 3.1 of J13. The model and radiative transfer calculations were made using the Juno atmospheric microwave radiative transfer (JAMRT, Janssen et al., 2005, in preparation) program, which is in development for the Juno Microwave Radiometer (MWR) experiment on Jupiter. To match the RADAR observations, radiative transfer calculations are carried out at $2.2-\mathrm{cm}$ wavelength $(13.78 \mathrm{GHz})$, and brightness temperatures are output for each observation. This model builds an atmosphere with user-prescribed physical parameters, such as the vertical mixing ratio profiles of ammonia, phosphine and water. Temperature and pressure profiles are calculated assuming hydrostatic equilibrium using both wet and dry adiabats. The reference model assumes a moist adiabatic temperature profile with $100 \%$ relative humidity $(\mathrm{RH})$, with a dry adiabatic profile below cloud base, such that the temperature is monotonically decreasing from the bottom to the top layer of the model atmosphere. The adiabats include the contributions from the $\mathrm{NH}_{4} \mathrm{SH}$ and $\mathrm{H}_{2} \mathrm{O}$ clouds, although the weighting function drops to essentially zero before we reach the water cloud at great depth. A temperature of $134.8 \mathrm{~K}$ (Lindal et al., 1985) is specified at a pressure of $1 \mathrm{bar}$, and the model temperature profile is slaved to this reference value. We varied this value in order to test the sensitivity of the $2.2-\mathrm{cm}$ brightness temperature to variations in the 1-bar temperature, and found the brightness temperature to be only minimally sensitive to this reference value (see Section 5.1). The topmost level of the model is the level at which the temperature reaches $110 \mathrm{~K}$, which is $560 \mathrm{mb}$ for the ( $134.8 \mathrm{~K}, 1 \mathrm{bar}$ ) reference point. The model assumes a completely transparent atmosphere above $110 \mathrm{~K}$ and therefore ignores this region of the atmosphere. The deepest level of the model atmosphere is 1000 bars, which is well below the pressure level sensed by the $2.2-\mathrm{cm}$ observations, and the vertical layers are $100 \mathrm{~m}$ thick. The model also includes the emission angle dependence (limb darkening) of the brightness temperature.

Table 1 gives the atmospheric constituents and their respective abundances in the model atmosphere, including the values used for the solar abundances. $\mathrm{H}_{2} \mathrm{O}, \mathrm{NH}_{3}, \mathrm{PH}_{3}$, and $\mathrm{H}_{2} \mathrm{~S}$ are the condensable gases (Atreya, 2010). $\mathrm{H}_{2} \mathrm{~S}$ reacts with $\mathrm{NH}_{3}$ to form an $\mathrm{NH}_{4} \mathrm{SH}$ cloud with a base around 5 bars. An ammonia ice cloud forms above this, with a base around 1.5 bars. The water cloud is deeper (base $\sim 10$ bars) and out of the sensitivity range of the $2.2-\mathrm{cm}$

Table 1

Abundances of atmospheric constituents in the JAMRT program. Solar and enrichment values are from Atreya (2010), who calculated solar abundances from the photospheric values of Grevesse et al. (2005).

\begin{tabular}{lll}
\hline Constituent & Solar abundance (relative to $\mathrm{H}_{2}$ ) & Enrichment relative to solar \\
\hline $\mathrm{He}$ & 0.195 & 0.6955 \\
$\mathrm{CH}_{4}$ & $5.50 \times 10^{-4}$ & 9.4 \\
$\mathrm{H}_{2} \mathrm{O}$ & $1.026 \times 10^{-3}$ & 3.0 \\
$\mathrm{NH}_{3}$ & $1.352 \times 10^{-4}$ & 3.0 \\
$\mathrm{H}_{2} \mathrm{~S}$ & $3.10 \times 10^{-5}$ & 5.0 \\
$\mathrm{Ar}$ & $7.24 \times 10^{-6}$ & 1.0 \\
$\mathrm{PH}_{3}$ & $5.14 \times 10^{-7}$ & 7.5 \\
\hline
\end{tabular}


weighting function. In the model, the presence of the ammonia ice cloud particles does not affect the $2.2-\mathrm{cm}$ brightness temperature significantly, although the depletion of ammonia vapor by the formation of the clouds does. Using Cassini Visible and Infrared Imaging Spectrometer (VIMS) data, Fletcher et al. (2011a) find evidence of a compact cloud deck in the 2.5-2.8 bar region. They point out that this is "deeper than the predicted condensation altitudes for pure $\mathrm{NH}_{3}$ clouds (1.47-1.81 bars) and higher than the predicted condensation altitudes for the $\mathrm{NH}_{4} \mathrm{SH}$ cloud (4.56-5.72 bars)." They conclude, "The VIMS 2.5-2.8 cloud cannot be identified unambiguously using the present data set." Resolving this discrepancy is beyond the scope of this paper, so we use an equilibrium condensation model similar to that referred by Fletcher et al. (2011a), even though it does not entirely fit their interpretation of the VIMS data. For a more in-depth description of the JAMRT program, see Section 3.1 of J13.

Because we only have data at one wavelength, there arises the unavoidable ambiguity of whether temperature or ammonia is the cause of the variations in brightness temperature. Our analysis assumes that atmospheric temperature is constant with latitude in the sensitivity range of 1-2 bars, and that variations in the ammonia mixing ratio cause brightness temperature variations. From a data-fitting point of view, one could also perform this analysis assuming that the mixing ratio of ammonia is constant with latitude and that atmospheric temperature fluctuations cause brightness temperature variations. In general, brightness variations can be due to both fluctuations in ammonia concentration and atmospheric temperature from latitude to latitude, and these are not easily separated. We think that ammonia dominating the brightness temperature variations at $2-\mathrm{cm}$ wavelength is the right choice, based on both the fact that emission from ammonia within the cloud region is strongly buffered against temperature variations, and also the extreme sensitivity of condensation/evaporation to vertical motions, as exhibited in the Earth's tropics. Large-scale subsidence, for example, will cause a "drying" of the atmosphere at certain latitudes, and could potentially produce a thermal emission pattern like the one we observe on Saturn. Our interpretation of the brightness temperature variations as variations in ammonia abundance is consistent with Grossman et al. (1989), who analyzed thermal emission from Saturn at 2- and 6-cm wavelength. They argue that large temperature deviations (on the order of $8 \mathrm{~K}$ in their paper) would be difficult to sustain in the presence of convection.

There are two basic ways to increase the brightness temperature $\mathrm{Tb}$. Either increase the atmospheric temperature $T$ with constant ammonia mixing ratio, or else hold $T$ constant and lower the ammonia mixing ratio. In both cases the relative humidity $(\mathrm{RH})$ goes down. If $\mathrm{RH}$ stays constant, an increase (decrease) in temperature is offset by an increase (decrease) in ammonia abundance due to the Clausius-Clapeyron relation, and the brightness temperature stays the same. Thus $\mathrm{Tb}$ is measuring $\mathrm{RH}$ in the sense that high RH gives low $\mathrm{Tb}$ and vice versa. When we refer to "drying out" of the atmosphere, we are referring to low RH acting to produce high $\mathrm{Tb}$.

We varied two parameters of the model to produce departures from the reference model: the enrichment factor of ammonia relative to the solar abundance (EF), and the ammonia depletion factor (DF). The enrichment factor EF is defined as the deep mixing ratio of ammonia vapor, expressed in terms of the solar abundance of ammonia (Table 1). In the model ammonia is uniformly mixed below the level where it reacts with $\mathrm{H}_{2} \mathrm{~S}$ to form an ammonium hydrosulfide cloud. It is partially depleted from that level up to the ammonia condensation level, where the mixing ratio of ammonia falls off according to the saturation vapor pressure dependence on temperature (the Clausius-Clapeyron relation). The deep abundance of ammonia is not precisely known on Saturn, but is thought to be in the range $2-4 \times$ solar (Atreya, 2010). We vary EF from 2 to
8 in this work (corresponding to volume mixing ratios of 2.3$\left.9.4 \times 10^{-4}\right)$. This range encompasses previous estimates for the deep abundance of gaseous ammonia, for example 4-6 $\times 10^{-4}$ reported by Briggs and Sackett (1989) and $5 \times 10^{-4}$ reported by de Pater and Massie (1985) from VLA measurements.

The depletion factor $\mathrm{DF}_{X}$ is chosen to allow an additional depletion of ammonia above some level $X$. Beginning with an ammonia mixing ratio distribution determined for an atmosphere with a given $\mathrm{EF}, \mathrm{DF}_{X}$ is simply a scale factor between 0 and 1 that multiplies the vertical distribution of ammonia above level $X$. It is intended purely as a first-order parameter to investigate ammonia depletions likely to occur in more realistic dynamical atmospheres. In applying $\mathrm{DF}_{X}$ we ignore any perturbations implied for other components of the model atmosphere such as the cloud base height or the temperature profile. For example, in the cloud layer, $\mathrm{DF}_{\mathrm{cb}}$ would correspond to the relative humidity ( $\mathrm{RH}$ ) of ammonia, where we choose the cloud base as our level $X$. In the following we choose $\mathrm{DF}_{\mathrm{cb}}$ and a second choice $\mathrm{DF}_{5 \mathrm{bar}}$, with level $X$ as the 5bar pressure level, to effectively bracket the cases we will study for ammonia depletion. We will show that both parameters are needed to explain the observed 2-cm brightness temperatures.

Figs. 1-3 demonstrate the effects of varying EF and DF in the model atmosphere. Fig. 1 shows two ammonia profiles, given by the heavy solid $(\mathrm{EF}=8)$ and dashed $(\mathrm{EF}=3)$ lines, both with $\mathrm{DF}=1$ (at all altitudes). The ammonia mixing ratios are less than 3 and $8 \times$ solar at the 2.5 -bar limit of Fig. 1 because the $\mathrm{NH}_{4} \mathrm{SH}$ cloud has a base around 5 bars and depletes the ammonia above. The light solid and dashed lines are the weighting functions that correspond to the $8 \times$ solar and $3 \times$ solar ammonia profiles, respectively. The weighting function for $3 \times$ solar extends deeper because there is less ammonia at the 1.5-1.8 bar level to block the radiation from below. The dotted curves are the temperature profiles for the two atmospheres, which differ only very slightly at the 2.5 bar level (with the $3 \times$ solar case being slightly warmer than the $8 \times$ solar case). The calculated brightness temperatures for these two models are $148.0 \mathrm{~K}$ for $3 \times$ solar and $147.9 \mathrm{~K}$ for $8 \times$ solar ammonia. Increasing $\mathrm{EF}$ further has very little effect on the brightness temperature.

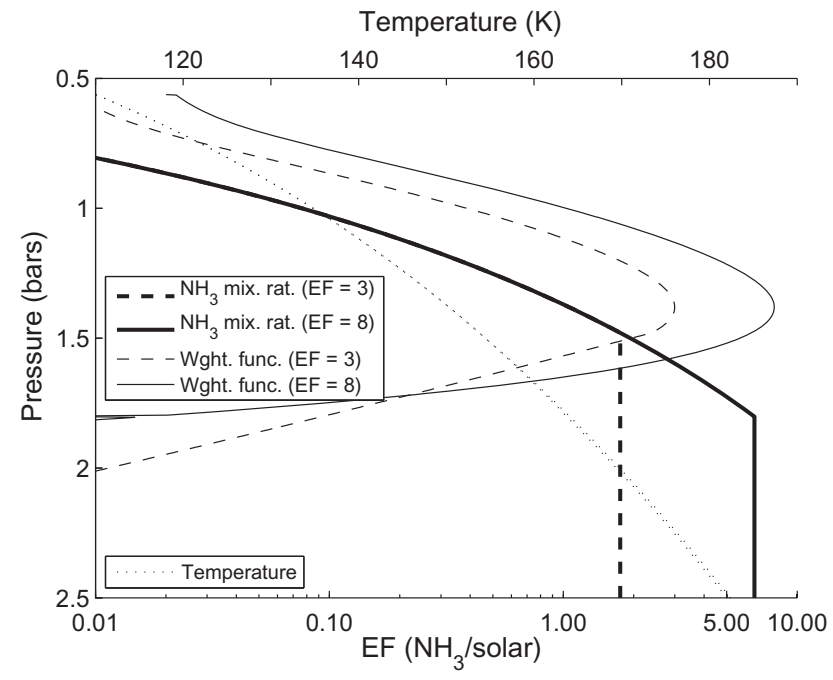

Fig. 1. Two vertical profiles of ammonia vapor with varying $\mathrm{EF}$ (bold lines). The solid and dashed bold lines are profiles with $\mathrm{EF}=8$ and 3, respectively, and the solid and dashed lines are their respective weighting functions. Their temperature profiles (top $x$-axis) are given by the dotted lines, which are almost identical in this pressure range, except that the $3 \times$ solar case is slightly warmer than the $8 \times$ solar case at 2.5 bars. The model brightness temperatures for the 3 and $8 \times$ solar cases are $148.0 \mathrm{~K}$ and $147.9 \mathrm{~K}$, respectively. 


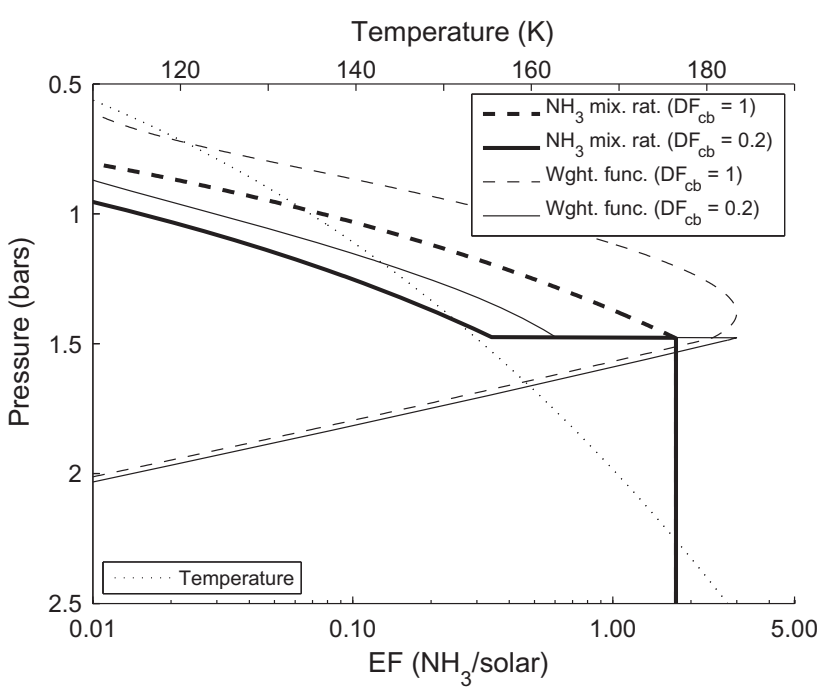

Fig. 2. Same as Fig. 1, but for constant $E F=3$ and varying $D F_{c b}$ (or ammonia $R H$ in the cloud layer). In this case the two model atmospheres have the same temperature profile. The case with $\mathrm{DF}_{\mathrm{cb}}=1$ is identical to the $\mathrm{EF}=3$ case in Fig. 1 (bold dashed line). There is a discontinuity in the ammonia mixing ratio at the cloud base for $\mathrm{DF}_{\mathrm{cb}}=0.2$ (bold solid line) due to the way the DF parameter functions in the model. The model brightness temperatures are $148.0 \mathrm{~K}$ and $154.0 \mathrm{~K}$ for the $\mathrm{DF}_{\mathrm{cb}}=1$ and 0.2 , respectively.

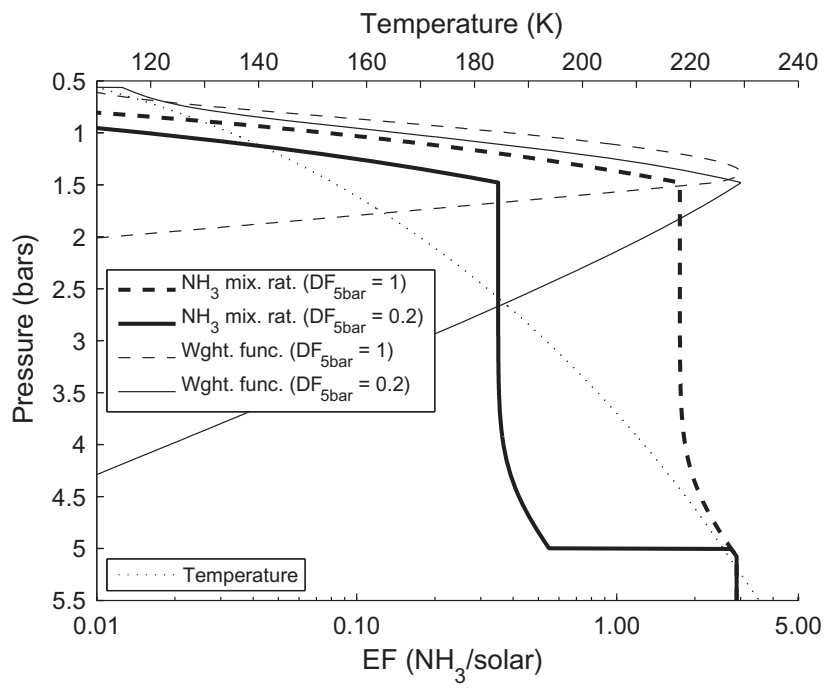

Fig. 3. Same as Figs. 1 and 2, but for constant $E F=3$ and varying $D_{5 b a r}$. Again, the $\mathrm{DF}_{5 \mathrm{bar}}=1$ case is the same as $\mathrm{EF}=3$ in Fig. 1 and $\mathrm{DF}_{\mathrm{cb}}=1$ in Fig. 2 . Note the change in scale of the $y$-axis. Like Fig. 2, there is a discontinuity in the ammonia mixing ratio at the level at which DF is applied (bold solid line), which is 5 bars for this case. The ammonia continues to be depleted between 4 and 5 bars because the formation of the $\mathrm{NH}_{4} \mathrm{SH}$ cloud at 5 bars causes ammonia depletion there. The model brightness temperatures are $148.0 \mathrm{~K}$ and $161.2 \mathrm{~K}$ for $\mathrm{DF}_{5 \mathrm{bar}}=1$ and 0.2 , respectively.

Fig. 2 is like Fig. 1 but with $\mathrm{EF}=3$ held constant and $\mathrm{DF}_{\mathrm{cb}}$ varied. The heavy solid and dashed lines are for atmospheric profiles of $\mathrm{DF}_{\mathrm{cb}}=0.2(\mathrm{RH}=20 \%)$ and $1(\mathrm{RH}=100 \%)$, respectively. The dotted line is the temperature profile of both atmospheres. The calculated brightness temperatures for $\mathrm{DF}_{\mathrm{cb}}=1$ and 0.2 are $148.0 \mathrm{~K}$ and $154.0 \mathrm{~K}$ respectively.

Fig. 3 is like Fig. 2, except $\mathrm{DF}_{5 \mathrm{bar}}$ is varied. Note the difference in scale of the $y$-axis between Fig. 3 and the previous two figures. In addition to the imposed depletion above the 5 bar level, the $\mathrm{NH}_{4} \mathrm{SH}$ cloud also depletes ammonia above the 5 bar level, which is why there is still some ammonia depletion above 5 bars for $\mathrm{DF}_{5 \mathrm{bar}}=1$. The calculated brightness temperatures for $\mathrm{DF}_{5 \mathrm{bar}}=1$ and 0.2 are $148.0 \mathrm{~K}$ and $161.2 \mathrm{~K}$, respectively.

The explanation for the differing brightness temperatures in each model atmosphere is as follows: If there is relatively less ammonia in a given atmospheric column (from the top down), then the $2.2-\mathrm{cm}$ weighting function will have contributions from higher pressures. Because the model temperature falls off adiabatically with height at all levels, the $2.2-\mathrm{cm}$ brightness temperature will be higher when there is less ammonia in the column, since we are probing a lower altitude in the atmosphere where the temperature is warmer. The opposite is true if there is relatively more ammonia in the column; namely, the resulting brightness temperature will be lower.

We chose to vary DF down to a level of 5 bars, which sparks the question: how deep is it necessary to deplete ammonia in order to achieve the observed brightness temperatures? Fig. 4 helps answer this question by displaying $\mathrm{DF}_{X}$ as a function of $X$, where $X$ is the pressure level to which we deplete ammonia ( $E F=3$ for all of these calculations). Except for the smallest values of $\mathrm{DF}_{X}$, the curves are flat for $X>2$ bars. Depleting down to 5 bars is equivalent to depleting down to 2 bars, which means that the $2.2-\mathrm{cm}$ weighting function is very small below 2 bars in this parameter regime $(E F=3$, $0.1 \leqslant \mathrm{DF}_{X} \leqslant 1$ ), and the brightness temperature is not very sensitive to depletion below the 2 bar level. For values of $\mathrm{DF}_{X}$ less than 0.1 , the brightness temperature is very sensitive to the depth of depletion because the weighting function peaks at $X$ bars for small $\mathrm{DF}_{\mathrm{X}}$. A similar plot was made for $\mathrm{EF}=6$, and the difference was that the brightness temperature of the $6 \times$ solar case was less than the $3 \times$ solar case by $1-7 \mathrm{~K}$ depending on the value of $\mathrm{DF}_{X}$. The lower brightness temperatures for $\mathrm{EF}=6$ are to be expected because when $\mathrm{EF}$ is larger, there is less emission from the deeper levels and the upper levels must be depleted a little more to get the same brightness temperature.

\section{Maps}

After using the radiative transfer model to calculate the brightness temperature as a function of emission angle, the residual brightness temperatures were calculated relative to a saturated atmosphere $(\mathrm{RH}=100 \%$ above the condensation level)

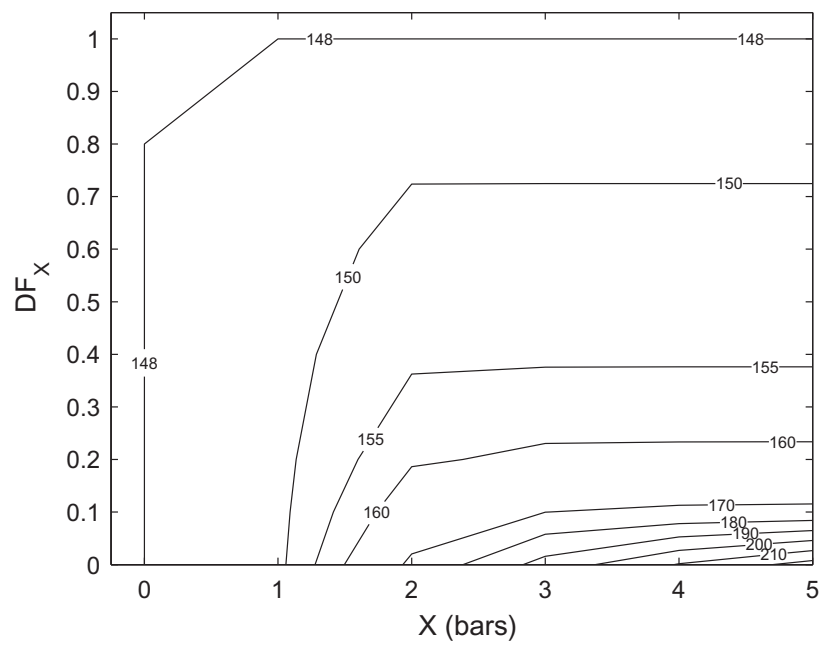

Fig. 4. Contours of model brightness temperature (in Kelvin) as a function of the depletion factor $\mathrm{DF}_{X}$ and $X$ (the depth of depletion) for constant enrichment factor of ammonia relative to the solar abundance $\mathrm{EF}=3 . \mathrm{DF}_{X}$ is a scalar parameter between 0 and 1 that multiplies the vertical distribution of ammonia above some pressure level $X$. Thus, $\mathrm{DF}_{X}=1$ is no depletion and $\mathrm{DF}_{X}=0$ is $100 \%$ depletion of ammonia vapor above level $X$. 
model with the constituent enrichments given in Table 1. All future mention of residual brightness temperatures refers to the residuals from this $3 \times$ solar ammonia reference model, which was chosen based on the ability of the $\mathrm{EF}=3$ reference model to span the observed brightness temperatures better than the models with higher or lower values of EF (Section 4, Figs. 11 and 12). Fig. 5 is a map from December 9, 2009 of the 2.2-cm residual brightness temperature, created by the procedure explained in Section 3.2 of J13. There are four other maps analyzed in this work but not shown here: September 23, 2005, October 13, 2009, July 25, 2010 and March 20, 2011. They are presented in Fig. 9 of J13.

\subsection{Obstruction by the rings}

An issue persistent throughout all the maps is that the equator is obstructed by the rings (black region along equator in Fig. 5). The rings are optically thick scatterers with very little intrinsic thermal emission, and hence lower the measured brightness temperature in the northern (southern) hemisphere when the spacecraft is below (above) the ring plane. When the spacecraft is in the ring plane, the ring inclination angle is exactly $0^{\circ}$ and the rings disappear from view, allowing full view of the equator. Fig. 6 demonstrates the effect of the ring obstruction on brightness temperature for the July 2010 map, the map for which the effect of the rings is most prominent (see Fig. 9 of J13). Each line is a different meridional (pole-to-pole) scan near the spacecraft's ring plane crossing (RPC), labeled with the ring inclination angle. For an example of a RPC scan see Fig. 5 near longitude $18^{\circ} \mathrm{W}$, where the ring blockage at the equator goes to zero. During the July 2010 observation period, Cassini was moving rapidly across the ring plane. Thus the effect of the rings on the brightness temperature is large, $50 \%$ between two scans taken only minutes apart (e.g. from scan $-0.019^{\circ}$ to $-0.377^{\circ}$ ). Fig. 6 demonstrates that ring inclination angles as small as $0.1^{\circ}$ have large effects on the observed equatorial brightness temperature.

Cassini crossed the ring plane in four of the five maps. We test our ability to remove the ring effect with a model that assumes an isothermal $(150 \mathrm{~K})$ brightness temperature for the atmosphere and a ring brightness temperature of $25 \mathrm{~K}$. With this model we synthesize the individual brightness scans taking into account the actual geometry of the spacecraft and the instrument. The right panel of Fig. 7 shows the results from this model for the four scans

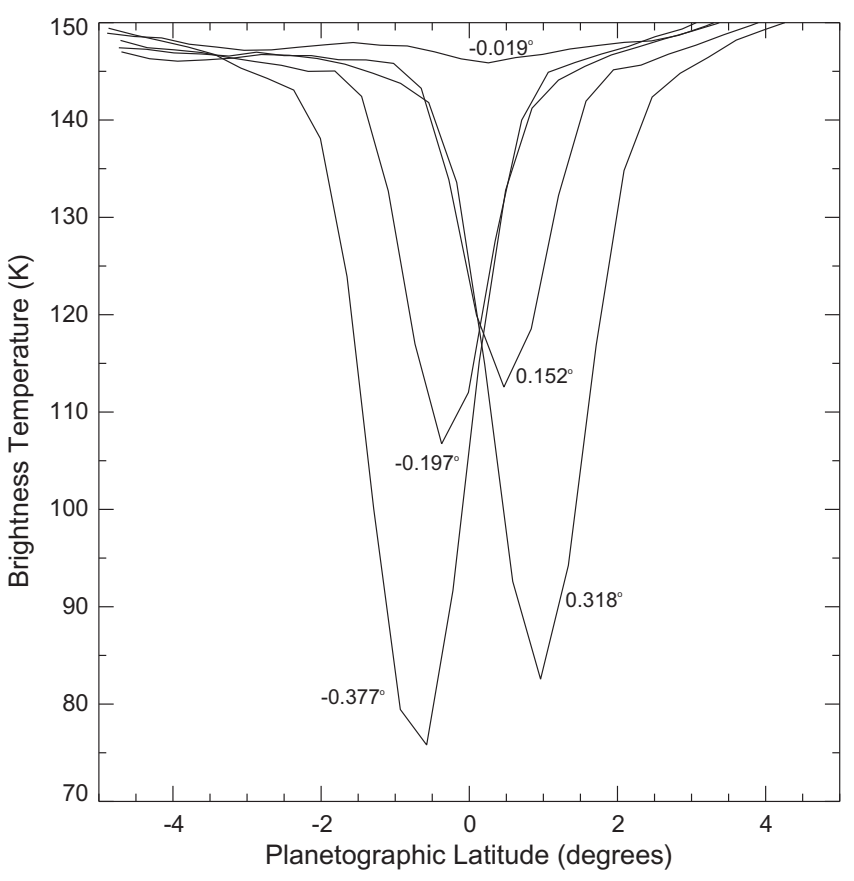

Fig. 6. Five individual scans around ring plane crossing (RPC) for the July 2010 map. Scans are labeled with their ring inclination angles as viewed by Cassini. The first scan, labeled $0.318^{\circ}$, was made while the spacecraft was in the southern hemisphere, therefore the ring blockage occurred in the northern hemisphere. As Cassini approached the ring plane, the effect of the rings became quite small. Because the spacecraft was moving fairly quickly across the ring plane in this map, the rings had a very large effect from one scan to the next.

closest to RPC for the December 2009 map, which has the smallest ring inclination angle while observing the equator. The labels are the same as in Fig. 6, but the scales along the $y$-axis are different. The left panel shows the observed brightness temperatures for the same scans. The model does not predict any dip in brightness temperature at the equator for the RPC scan $\left(0.001^{\circ}\right.$ ring inclination angle), demonstrating that we are observing the true equatorial brightness temperature in this scan. In the left panel, the $0.001^{\circ}$ scan is continuously flat through the equator (within $\pm 2^{\circ}$ ), which does not occur for any other RPC scan except for the

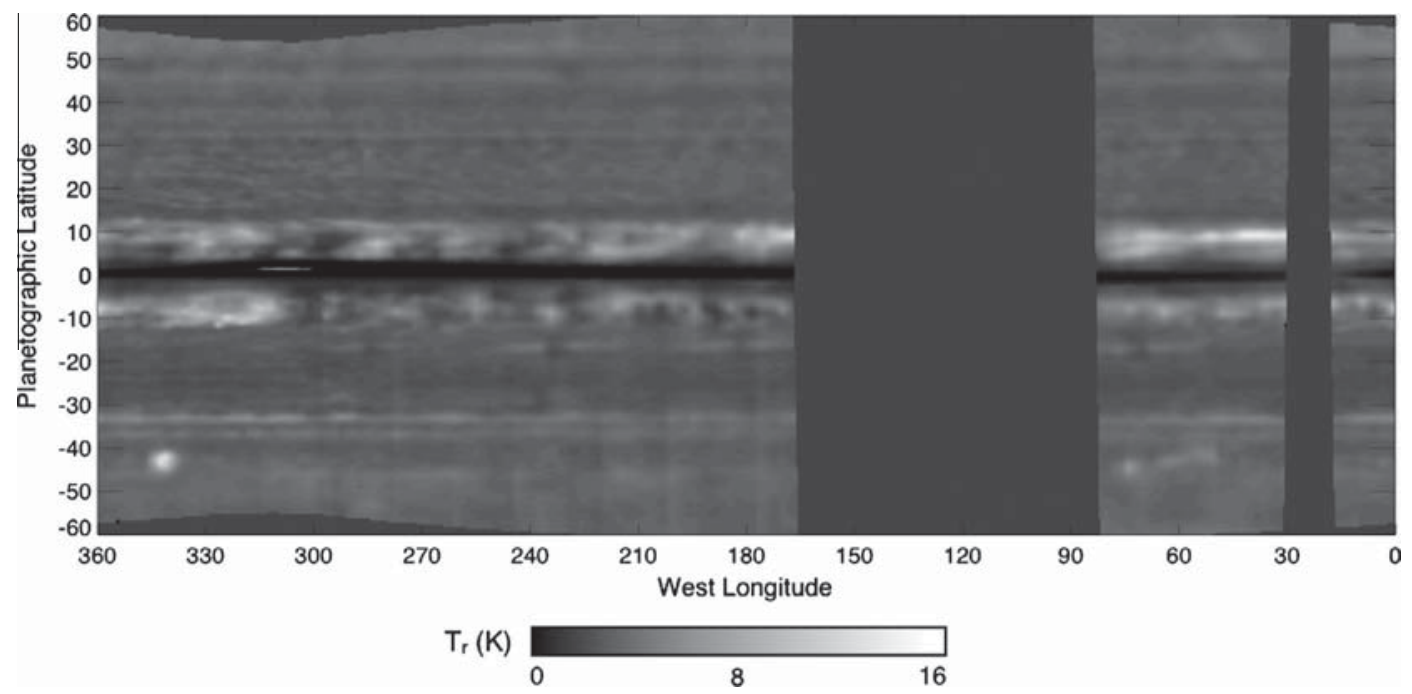

Fig. 5. 2.2-cm residual brightness temperature (in Kelvin) map of Saturn from December 9, 2009. The residual temperature is calculated by subtracting the brightness temperature from a fully-saturated reference model with $3 \times$ solar ammonia mixing ratio (Section 2 ) from the observed brightness temperature. The black band at the equator is due to the cold rings obstructing the atmosphere. Section 3.2 of J13 offers a detailed explanation of how the brightness temperature maps were generated. 

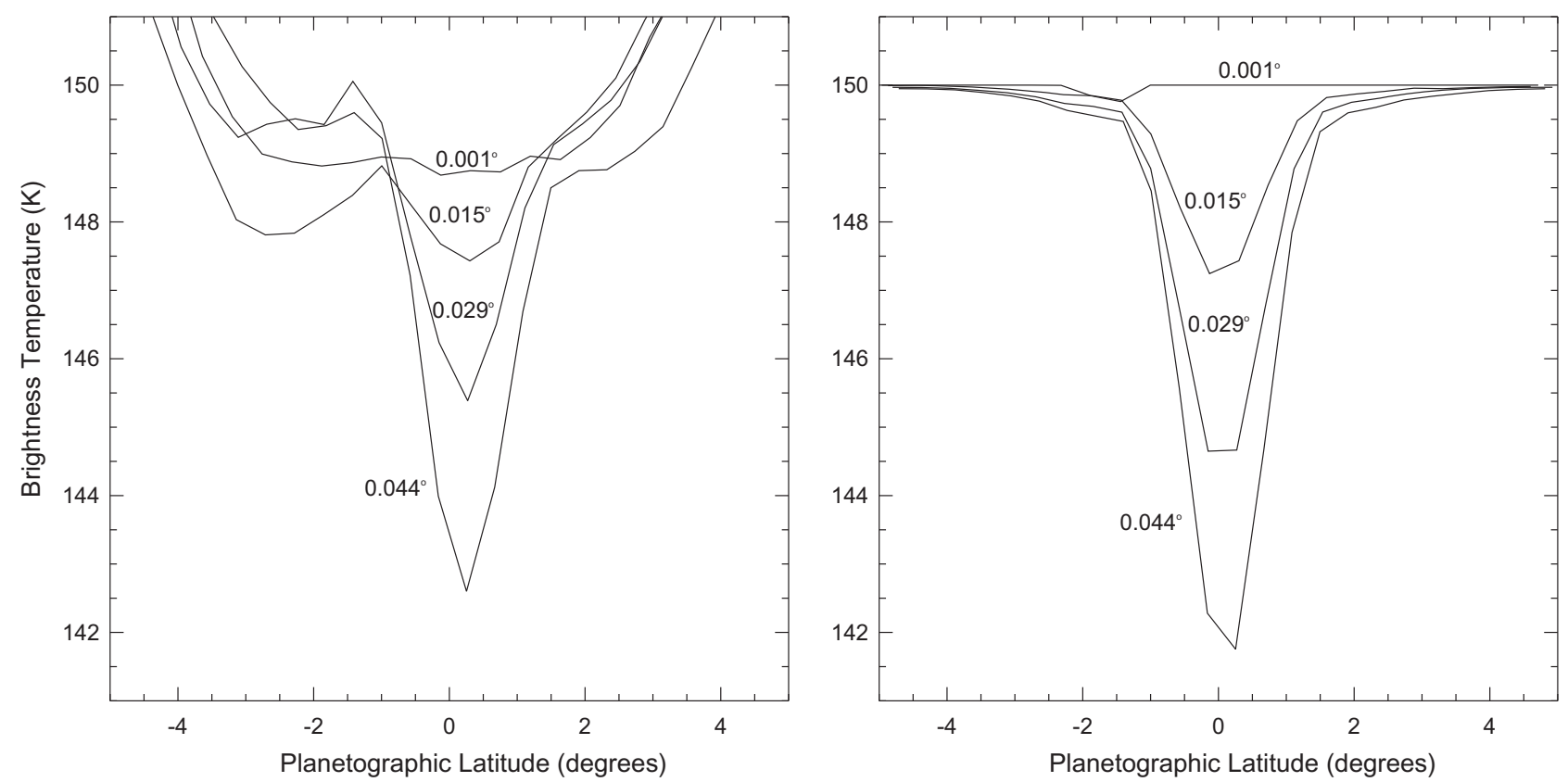

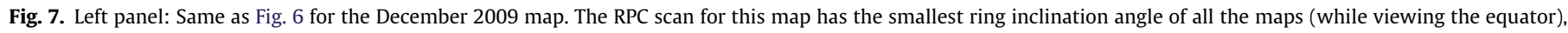
and is flat across the equator. This is the best view of the equatorial brightness temperature that we have of all five maps. Right panel: Same labeling, but for a simple beam convolution model that takes into account the Cassini-Saturn geometry and includes the A and B rings only. It assumes an isothermal atmosphere of $150 \mathrm{~K}$, and takes the microwave brightness of both the A and B rings to be $25 \mathrm{~K}$. According to this model, we actually see the equator with no ring blockage for the $0.001^{\circ}$ scan.

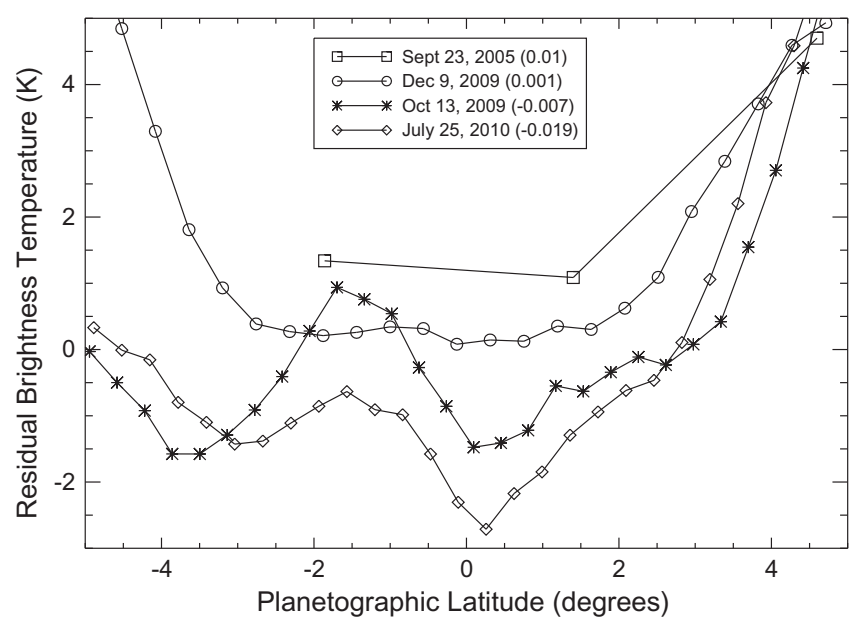

Fig. 8. Residual brightness temperatures for RPC scans of the four maps and their average. The ring inclination angle for each scan is in parentheses next to the date. Because of the low resolution of the September 2005 scan, the bright bands off the equator affect the equatorial brightness temperature, causing it to be $0.7 \mathrm{~K}$ too high. With this in mind, the four scans are within $\pm 1.5 \mathrm{~K}$. The December $2009 \mathrm{RPC}$ scan is flat across the equator with a very small ring inclination angle. Thus it provides the true equatorial brightness temperature.

September 2005 scan, which is at very low resolution compared with the other three maps (Fig. 8). Thus we take the December RPC scan as the RPC scan that provides the true equatorial brightness temperature. The increase in brightness temperature at latitudes greater than $3^{\circ}$ in both hemispheres is not in the model but is a real property of Saturn's atmosphere.

Fig. 8 displays residual brightness temperatures versus latitude for all four RPC scans. The scale along the $y$-axis is expanded relative to that in Figs. 6 and 7. The ring inclination angles for each scan are displayed in parentheses in the legend. The segment of the

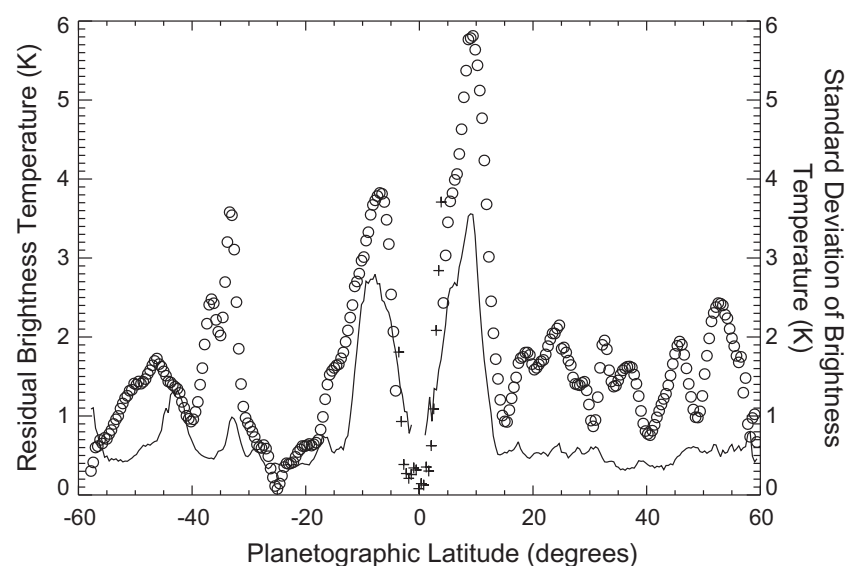

Fig. 9. Mean residual brightness temperature (open circle and + signs) and its mean standard deviation as a function of planetographic latitude (solid line) for all five maps, excluding the northern storm and the latitudes near the equator where the rings block the view of the atmosphere. Observations were sorted into latitude bins $0.4^{\circ}$ wide since the latitudes are unevenly spaced. From $-4^{\circ}$ to $+4^{\circ}$ the single December 2009 RPC scan is used (+ signs), because it is the best view of the equatorial brightness temperature that we have of all the maps (Section 3.1). Standard deviations were calculated at each latitude for each map and then averaged. The average is weighted by the number of observations at a given latitude and date and the sum is over all five observation dates.

September 2005 map where the RPC occurred is at very low resolution (Fig. 9, J13), and is therefore affected by the two bright bands at $\pm 9^{\circ}$ with a contribution of about $+0.7 \mathrm{~K}$. Applying this correction to the September 2005 scan brings the residual brightness temperature down to $0.5 \mathrm{~K}$ at the equator. The observed residuals are consistent with $\sim 1 \mathrm{~K}$ variability in the equatorial region. We chose the December 2009 RPC scan to represent the equatorial brightness temperature for all five maps in Fig. 9 because it has the best geometry (lowest inclination at RPC) and produces the best picture of the equatorial brightness temperature that we have. 


\subsection{General features}

With the exception of the March 2011 map, which has the 2010-2011 northern storm (Figs. 9 and 12, J13) or great white spot (Fischer et al., 2011; Sánchez-Lavega et al., 2011), all of the maps share the same general characteristics. In what follows, all latitudes are planetographic unless explicitly stated. In Fig. 5, the equatorial region, within $10^{\circ}$ of the equator, is texturally anomalous compared with the rest of the map, even when excluding the effect of the rings. There is non-uniform high brightness near $-9^{\circ}$ and $9^{\circ}$ that generally decreases towards the equator, which is obstructed by the rings for all scans except for the one during RPC. The brightness temperature variations on the maps are quite large, with variations of more than $10 \mathrm{~K}$, and we investigate the causes of these variations. There are some structures in the southern hemisphere band between $-42^{\circ}$ and $-47^{\circ}$ (e.g. at $340^{\circ} \mathrm{W}$ and $75^{\circ} \mathrm{W}$ in Fig. 5), which is just south of the westward jet at $-42^{\circ}$ $\left(-35^{\circ}\right.$ planetocentric) and has been the site of many lightning observations (Dyudina et al., 2007; Fischer et al., 2011). There are also two narrow bright bands at $-33^{\circ}$ and $-37^{\circ}$, and a broad dark band from $-15^{\circ}$ to $-30^{\circ}$.

The highest brightness temperature in the northern storm is $165.7 \mathrm{~K}$ ( $18.9 \mathrm{~K}$ residual brightness temperature). The highest brightness temperature in all five maps is $167.1 \mathrm{~K}(19.3 \mathrm{~K}$ residual brightness temperature), which occurs in the subtropical latitudes in the October 2009 map.

Fig. 9 shows the zonally averaged residual brightness temperature (open circles and + signs) and its standard deviation (solid line) as a function of latitude. Outside of $\pm 4^{\circ}$ latitude, the brightness temperatures are averaged over longitude and over all five maps (open circles), excluding the 2010-2011 northern storm and the latitudes near the equator where the rings block the view of the atmosphere. Within $\pm 4^{\circ}$ of the equator, a single RPC scan from the December 2009 map is used (+ symbols), as explained in Section 3.1. The standard deviation was computed with respect to longitude for each latitude outside $\pm 4^{\circ}$, and then the weighted standard deviation for all five maps was calculated. The weights used in this calculation are the number of observations at a given latitude and date and the sum is over all five observation dates. The globally averaged residual brightness temperature from Fig. 9 is $1.7 \pm 1.1 \mathrm{~K}$, where the $1.1 \mathrm{~K}$ is real variability of the longitudinally-averaged brightnesses in Saturn's atmosphere.

The residual brightness temperatures shown in Fig. 9 are positive at every latitude. One important implication of this observation is that the atmosphere is always ammonia-depleted with respect to a fully-saturated $3 \times$ solar ammonia model (Table 1 ). A striking feature of Fig. 9 is the two relatively bright bands near $\pm 9^{\circ}$, the subtropics of Saturn, with residuals of $3.8 \mathrm{~K}$ and $5.8 \mathrm{~K}$ in the southern and northern hemispheres, respectively (corresponding to brightness temperatures of $151.7 \mathrm{~K}$ and $153.7 \mathrm{~K}$ ). These two bright latitudes surround a relatively low residual brightness temperature of $0.1 \mathrm{~K}$ (corresponding to a brightness temperature of $148.1 \mathrm{~K}$ ) at the equator. At the equator the atmosphere is close to being saturated with ammonia. The subtropical bands are accompanied by elevated standard deviation, indicating that there is some structure in these regions. Another feature is the pair of bright bands around $-36^{\circ}$ and $-34^{\circ}$ with averaged residuals of 2.5 and $3.6 \mathrm{~K}$ respectively (corresponding to brightness temperatures of 149.2 and $150.5 \mathrm{~K}$ ), which correspond to the two narrow bands in the southern hemisphere in Fig. 5. There are two regions with high standard deviation in the southern hemisphere, one near $-33^{\circ}\left(-28^{\circ}\right.$ planetocentric) and the other near $-43^{\circ}\left(-37^{\circ}\right.$ planetocentric). The latter is the latitude of storm alley and the site of the southern hemisphere lightning (Dyudina et al., 2007). The relatively high standard deviation in storm alley corresponds to the bright dots seen there (Fig. 5 , longitude $345^{\circ} \mathrm{W}$ ), which are likely to be holes in the ammonia layer associated with the holes in the clouds described by Dyudina et al. (2007). Dyudina et al. (in preparation) investigates the structure of these southern hemisphere storms and presents the lightning observations from both the southern storms and the 2010-2011 northern storm. The northern latitudes have relatively constant brightness temperatures, with fluctuations on the order of $1 \mathrm{~K}$ from latitude to latitude. The southern hemisphere has larger brightness temperature gradients than the northern hemisphere, for example almost a $4 \mathrm{~K}$ increase from $-25^{\circ}$ to $-35^{\circ}$. For the northern storm (not included in Fig. 9), the standard deviation in the latitude band between $20^{\circ}$ and $50^{\circ}$ is much larger, reaching a peak value of $6 \mathrm{~K}$ at $40^{\circ}$.

When looking at the relatively bright spots within the bright subtropical bands in all five maps, it is natural to ask if there is any periodic structure in these regions. Jupiter, for example, had the equatorial plumes in the northern hemisphere with longitudinal wavenumber between 11 and 13 at the time of the Voyager encounters (Allison, 1990), and between 8 and 12 determined more recently (Arregi et al., 2006). To determine whether there is periodic structure in the subtropical bands of Saturn, we calculated the autocorrelation of the brightness temperature with respect to longitude for latitude bands between $6^{\circ}$ and $10^{\circ}$ in both hemispheres. We used binning to remedy the problems that arise due to unevenly spaced observation points and large gaps in the maps. For a given latitude, every pair of points was placed into a bin $3^{\circ}$ wide based on the lag between the points. Thus the $0^{\circ}$ lag bin has pairs of points with lags from $0^{\circ}$ to $3^{\circ}$. The next bin is $3-6^{\circ}$, and so on. We did this for four maps, excluding the July 2010 map because the rings obstruct the majority of the subtropical bands. The resulting averaged correlation coefficients are plotted versus longitudinal lag in Fig. 10. The correlations in the southern hemisphere tend to fall off more rapidly than in the northern hemisphere, with the exception of the December 2009 map. This indicates that the bright spots in the northern hemisphere subtropical band have a greater longitudinal span than those in the southern hemisphere. Four of the eight panels in Fig. 10 reveal wave-like features with longitudinal periods ranging from $20^{\circ}$ to $45^{\circ}$ (zonal wavenumbers $18-8$, respectively) for the $6-10^{\circ}$ latitude range. The period varies from year to year, and there is no indication of a recurring dominant period. We did not find any wave-like features in other latitude ranges.

\section{Comparison with radiative transfer model}

Ammonia vapor is the only effective source of opacity in Saturn's atmosphere at $2.2-\mathrm{cm}$ wavelength, as shown by Fig. 3 of $\mathrm{J} 13$ that plots absorption coefficient versus height for the relevant constituents. Ammonia is by far the dominant absorber in our sensitivity range. Thus the $2.2-\mathrm{cm}$ brightness temperature maps yield information about the ammonia vapor distribution. To interpret the maps we used the JAMRT program described in Section 2 to calculate $2.2-\mathrm{cm}$ brightness temperatures based on different vertical profiles of ammonia. We varied two parameters, EF and DF, also described in Section 2.

Figs. 1 and 2 suggest that we cannot get brightness temperature variations larger than $11 \mathrm{~K}$ by varying only $\mathrm{EF}$ (ammonia deep abundance) or $\mathrm{DF}_{\mathrm{cb}}$ (ammonia $\mathrm{RH}$ in the cloud layer), since these two parameters only account for brightness temperature variations of up to $11 \mathrm{~K}$. Fig. 11 demonstrates this by displaying the model brightness temperature (in Kelvin) as a function of $\mathrm{DF}_{\mathrm{cb}}$ (or RH) and EF. All the calculations done in Figs. 11 and 12 were done at an emission angle of $0^{\circ}$. This is important to note because the model brightness temperatures decrease with increasing emission angle due to limb darkening. For example, a brightness temperature of $160 \mathrm{~K}$ at the equator would have a residual of $12 \mathrm{~K}$, while a brightness temperature of $160 \mathrm{~K}$ at $40^{\circ}$ (e.g., the 2010 2011 northern storm) would have a residual of $13.5 \mathrm{~K}$. 

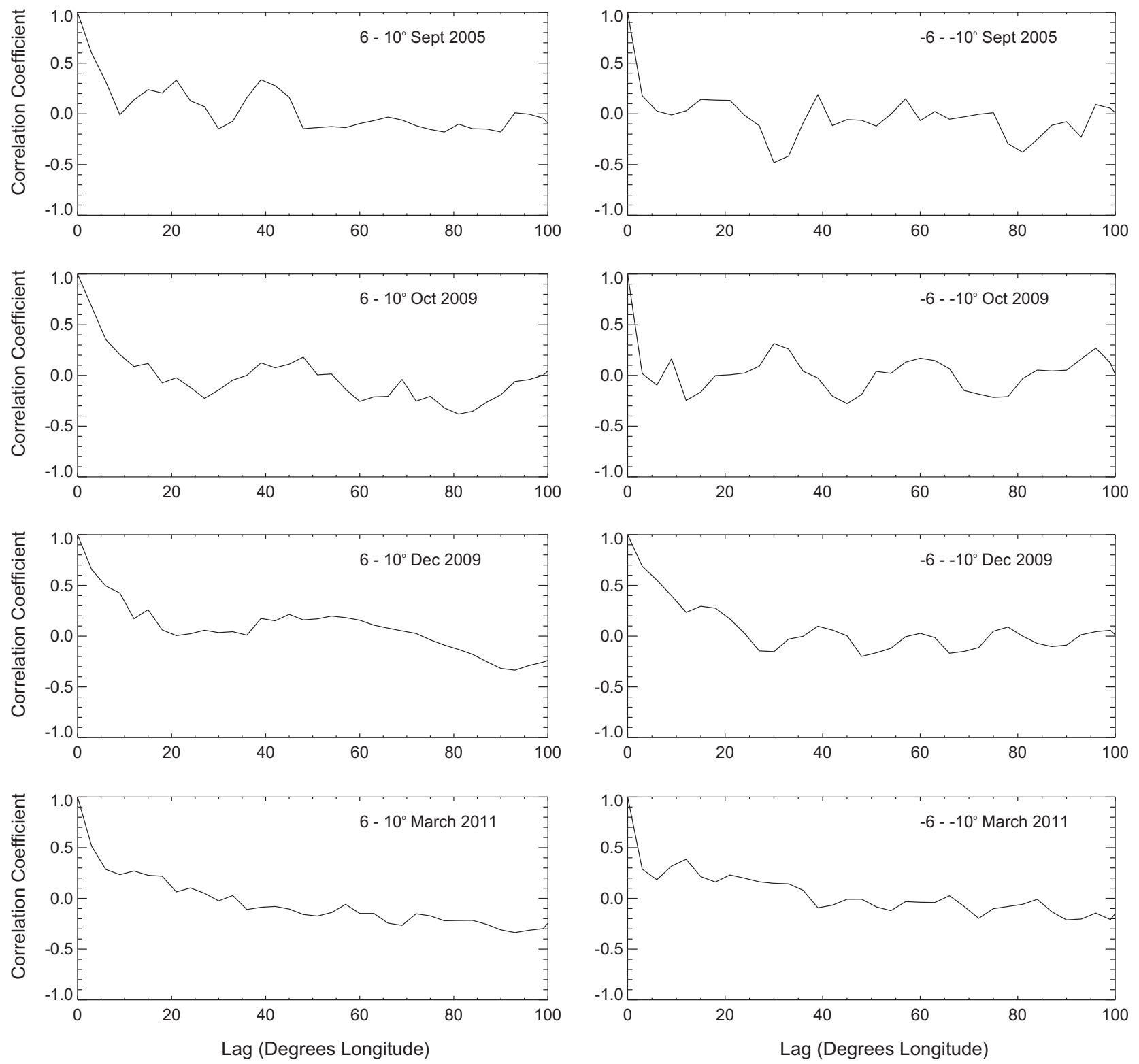

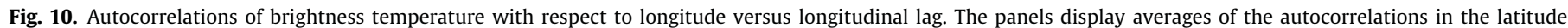

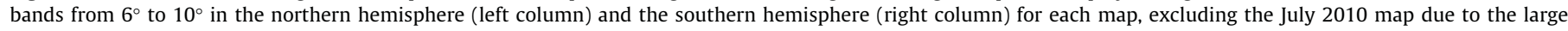
amount of ring blockage in the subtropics.

In Fig. 11, as expected, the brightness temperatures increase with decreasing $\mathrm{DF}_{\mathrm{cb}}$ (decreasing $\mathrm{RH}$ ), since depletion of ammonia pushes the weighting function deeper into the atmosphere where it is warmer. However, as mentioned above, the largest temperature contrast that can be obtained in this parameter regime is $11 \mathrm{~K}$ (148-159 K, Fig. 11), which indicates that ammonia vapor must be depleted to levels deeper than the ammonia cloud in order to achieve the highest brightness temperatures seen in the maps $(160+\mathrm{K})$, provided that $\mathrm{DF}_{\mathrm{cb}}>0.1$. If $\mathrm{DF}_{\mathrm{cb}}<0.1$, then the ammonia concentration in the cloud region is almost zero, which is unlikely given ammonia concentrations reported by Fletcher et al. (2011a) for the 1-4 bar region. The idea that the formation of the $\mathrm{NH}_{4} \mathrm{SH}$ cloud around 5 bars is the ammonia-depletion mechanism beneath the ammonia-ice cloud has been discussed by previous authors (e.g. Briggs and Sackett, 1989). Since our model includes the formation of this cloud (with $\mathrm{H}_{2} \mathrm{~S}$ enriched by $5 \times$ solar, Table 1 ), ammonia must be depleted even more than just by the $\mathrm{NH}_{4} \mathrm{SH}$ cloud formation in order to agree with observations, unless $\mathrm{H}_{2} \mathrm{~S}$ is in reality enriched by more than $5 \times$ solar on Saturn.
There is a trend of increasing brightness with increasing EF at low $\mathrm{DF}_{\mathrm{cb}}$ in Fig. 11. This is counter-intuitive, because increasing EF means more ammonia, but it is an artifact of the model and has an explanation. The $\mathrm{DF}_{\mathrm{cb}}$ parameter depletes ammonia down to the ammonia cloud base, and for very low $\mathrm{DF}_{\mathrm{cb}}$ that is where the weighting function peaks. As EF increases, the ammonia cloud base moves to deeper (and warmer) levels (Fig. 1), bringing the weighting function with it. The effect of lowering the altitude of the weighting function outweighs the effect of adding ammonia at deeper levels, and brightness temperature increases with increasing EF.

Fig. 12 is the same as Fig. 11 , but for $\mathrm{DF}_{5 \mathrm{bar}}$. Note that the contour interval has been increased from 1 to $4 \mathrm{~K}$. As expected, the brightness temperature increases with decreasing $\mathrm{DF}_{5 \mathrm{bar}}$ and decreasing EF. By depleting the ammonia down to this deeper level ( 5 bars), we are able to achieve the $166-167 \mathrm{~K}$ brightness temperatures seen in the maps. This requires either $\mathrm{DF}_{\mathrm{cb}}<0.1$ (Fig. 11, lower right portion) or a combination of $\mathrm{DF}_{5 \mathrm{bar}}<0.3$ and $\mathrm{EF}<4$ (Fig. 12, lower left corner). Figs. 11 and 12 are for rays propagating 


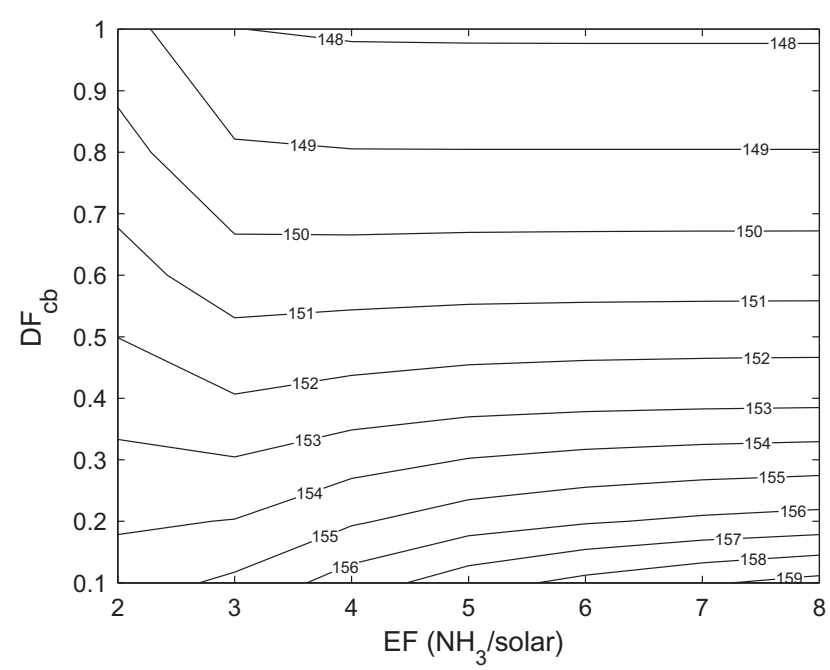

Fig. 11. Contours of model brightness temperature (in Kelvin) as a function of the ammonia depletion factor above the cloud base height, $\mathrm{DF}_{\mathrm{cb}}$ (or $\mathrm{RH}$ ), and the enrichment factor of ammonia relative to the solar abundance, EF. The warming trend at high EF is related to the way the cloud base changes with this parameter (see text for a more detailed explanation).

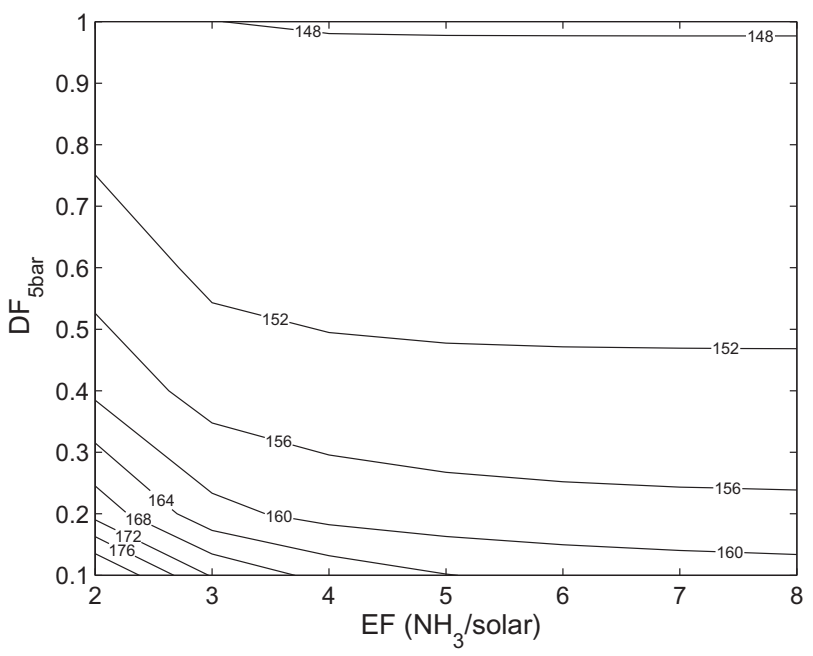

Fig. 12. Same as Fig. 11, but for the depletion factor above 5 bars, $D_{5 b a r}$. Note the contour interval has been increased to $4 \mathrm{~K}$.

vertically. Taking the limb darkening effect at $40^{\circ}$ latitude into account means that brightness temperatures in the storm correspond to $\mathrm{DF}_{5 \mathrm{bar}}=0.1$ for $\mathrm{EF}=3$, which is a very large depletion $(90 \%)$. The subtropical bright bands can be explained by either very low $\mathrm{DF}_{\mathrm{cb}}$ or mid-range values of $\mathrm{DF}_{5 \mathrm{bar}}$.

From our results it seems that ammonia lies near the $3 \times$ solar range, because at high $\mathrm{EF}$ values $(\mathrm{EF}>4)$ it becomes impossible to achieve the highest observed brightness temperature (167 K) unless $\mathrm{DF}_{5 \mathrm{bar}}<0.1$ (Fig. 12). Such low values of DF are incompatible with other observations of ammonia (i.e. Fletcher et al., 2011a). Also, at low EF values ( $\mathrm{EF}<3)$ it becomes impossible to achieve the lowest observed brightness temperature ( $148 \mathrm{~K}$ at the equator) unless the atmosphere there is supersaturated. This is because saturation occurs at $T>148 \mathrm{~K}$ for $\mathrm{EF}<3$ (Figs. 11 and 12, top left corner). According to this analysis, Saturn's atmosphere lies in the 3-4× solar ammonia range, with fairly large depletion of ammonia extending below the cloud base in some regions.

Since the highest brightness temperature seen in all five maps is near $167 \mathrm{~K}$ (in the subtropical latitudes of the October 2009 map), depleting down to 2 bars could explain all the brightness temperatures we see (Fig. 4). In this case $\mathrm{DF}_{2 \mathrm{bar}}$ would have to be close to 0 , which corresponds to no ammonia above 2 bars. This is not a likely scenario, so we focus on $\mathrm{DF}_{5 \mathrm{bar}}$ for the remainder of the paper, keeping in mind that $\mathrm{DF}_{5 \mathrm{bar}}$ is the same as $\mathrm{DF}_{3 \mathrm{bar}}$ or $\mathrm{DF}_{4 \mathrm{bar}}$ as long as $\mathrm{DF} \geqslant 0.2$ (Fig. 4).

\section{Discussion}

The deep abundance of heavy elements such as nitrogen and carbon is not well known on Saturn. Estimates range from 2 to $4 \times$ solar (Atreya, 2010) for ammonia to $9-10 \times$ solar (Fletcher et al., 2012) for carbon. The solar values used in these estimates are those given in Table 1, and they are given with respect to $\mathrm{H}_{2}$. Because we only have data at one wavelength, we cannot constrain EF and DF separately, we can only comment on possible combinations of the two parameters that give brightness temperatures consistent with the $2.2-\mathrm{cm}$ data. However, as presented above, EF must lie in the $3-4 \times$ solar range in order to achieve the highest and lowest brightness temperatures observed at $2.2-\mathrm{cm}$. For the following discussion, we assume that $\mathrm{EF}=3$ and then we comment on the values of DF that would yield the observed brightness temperatures. Note that $\mathrm{EF}=3$, which corresponds to a deep volume mixing ratio of $3.6 \times 10^{-4}$, is fairly consistent with values reported by de Pater and Massie (1985) and Briggs and Sackett (1989), whose estimates are for pressure levels greater than 3 bars and the 2-bar level, respectively, and Fletcher et al. (2011a) for the 14 bar range at the equator. Our estimate is also consistent with the estimate of $1.2 \times 10^{-4}$ obtained from VLA measurements by Grossman et al. (1989) for the condensation altitude.

\subsection{All maps}

Using the quantitative analysis from the radiative transfer model for $3 \times$ solar ammonia, we were able to convert the residual brightness temperatures to $\mathrm{DF}_{\mathrm{cb}}$ values, or values of ammonia $\mathrm{RH}$ in the ammonia cloud layer. Fig. 13a shows a map of the ammonia RH for the March 2011 observation date, with the northern storm being the most prominent feature. Black regions would be regions where the atmosphere is supersaturated with respect to ammonia in the cloud layer (we do not see any). In this figure black corresponds to where the atmosphere looks very cold due to the ring obstruction. Blue regions are regions that require depletion of ammonia below the clouds in order to achieve those high brightness temperatures.

Fig. 9 shows that the global average residual brightness temperature relative to the saturated model is $1.7 \pm 1.1 \mathrm{~K}$, where the $\pm 1.1 \mathrm{~K}$ is the real variability of longitudinally-averaged brightnesses in the atmosphere. Figs. 4, 11 and 12 give DF for various brightness temperature values, and the curves are almost flat for $X \geqslant 2$ bars (Fig. 4). In Fig. 11, each DF value is the RH at the corresponding brightness temperature. Taking brightness temperature $\mathrm{Tb}=148 \mathrm{~K}$ as the saturated case and $\mathrm{Tb}=149.7 \pm 1.1 \mathrm{~K}$ as the global average (for $\mathrm{EF}=3$ ), we find that the average $\mathrm{RH}$ is $70 \pm 15 \%$ in the cloud layer. Note that this estimate does not include the 2010-2011 northern storm, and that the $\pm 15 \%$ comes directly from the $1.1 \mathrm{~K}$ variability in Fig. 9. For comparison, de Pater et al. (2001) find that the disk-averaged relative humidity of ammonia in Jupiter's atmosphere is of the order of $10 \%$ at pressures less than 0.55 bars.

Saturn's atmosphere also has local and regional features with $\mathrm{RH}<0$ in the cloud layer. These regions are shown in green-blue in Fig. 13. It is clear that the northern storm contains some rich dynamics that cause ammonia depletion below the cloud base. We discuss the northern storm in more detail Section 5.2. The subtropical bands at $\pm 9^{\circ}$ have a lot of structure, with alternating 


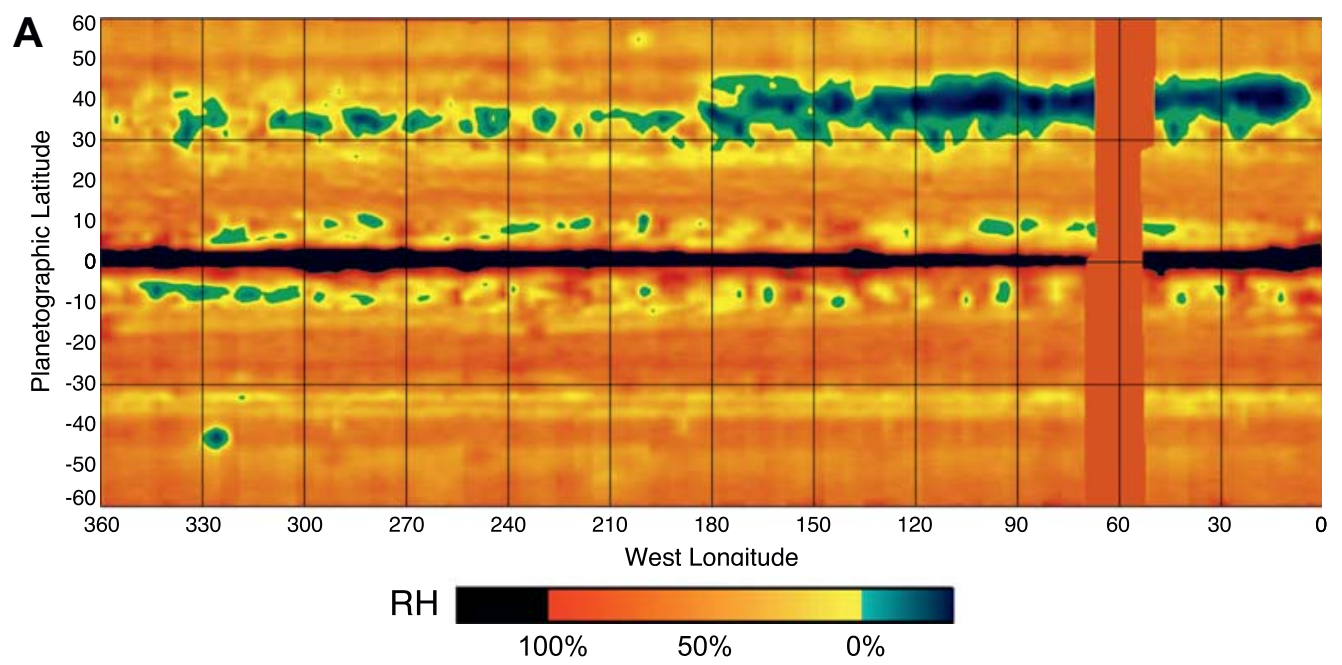

B
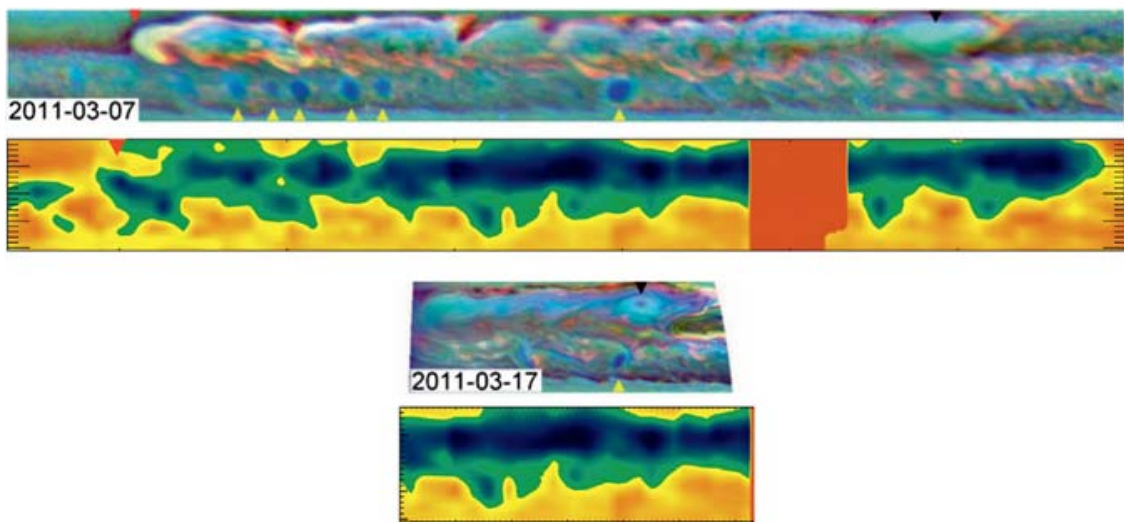

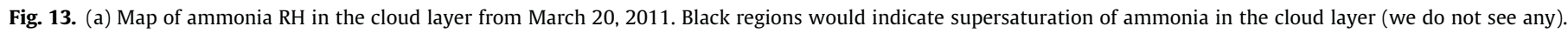
Here, the black regions are due to the cold rings blocking the emission from the atmosphere. Green to blue regions are regions that require ammonia depletion below the ammonia cloud layer (i.e. $\mathrm{RH}<0$ in the cloud layer). The northern storm is blue, indicating low ammonia concentrations in the storm that extend to layers beneath the clouds.

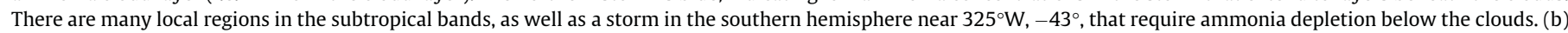

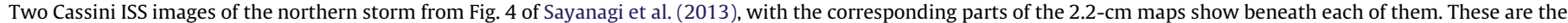

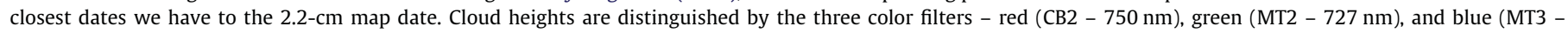
$889 \mathrm{~nm}$ ). (For interpretation of the references to color in this figure legend, the reader is referred to the web version of this article.)

regions of high and low ammonia $\mathrm{RH}$. In every map, there are local regions in the two subtropical bands that require ammonia depletion below the cloud base. As seen by the red-orange color in the figure, the band from $-15^{\circ}$ to $-30^{\circ}$ is more humid than other latitudes. The narrow bands at $-33^{\circ}$ and $-37^{\circ}$ are drier than their surrounding latitudes. South of these bands, there is a storm near $325^{\circ} \mathrm{W},-45^{\circ}$ that requires ammonia depletion below the clouds.

For comparison, we look at ammonia abundances reported by Fletcher et al. (2011a). Their values were derived from CassiniVIMS 4.6-5.1 $\mu \mathrm{m}$ spectra taken in April 2006. In Fig. 14i of their paper, the ammonia mole fraction is given as a function of latitude for their sensitivity range of 1-4 bars. There is a drastic difference between the equator and just off the equator in both hemispheres, with high ammonia abundance centered on the equator and extending to about $\pm 5^{\circ}$ planetocentric $\left( \pm 6^{\circ}\right.$ planetographic), and relatively low abundance at $\pm 10^{\circ}$ planetocentric $\left( \pm 12^{\circ}\right.$ planetographic). Our Fig. 9 is qualitatively consistent with these results-there is high ammonia at the equator (low 2.2-cm brightness temperature) and low ammonia in the subtropical bands. However, our figure suggests that there would be larger dips in the ammonia abundance in the subtropical bands than is shown in Fletcher et al. (2011a). Their Fig. 14i shows $\mathrm{NH}_{3}$ mole fractions of $450 \mathrm{ppm}$ at the equator, $110 \mathrm{ppm}$ at $\pm 8-10^{\circ}$, and

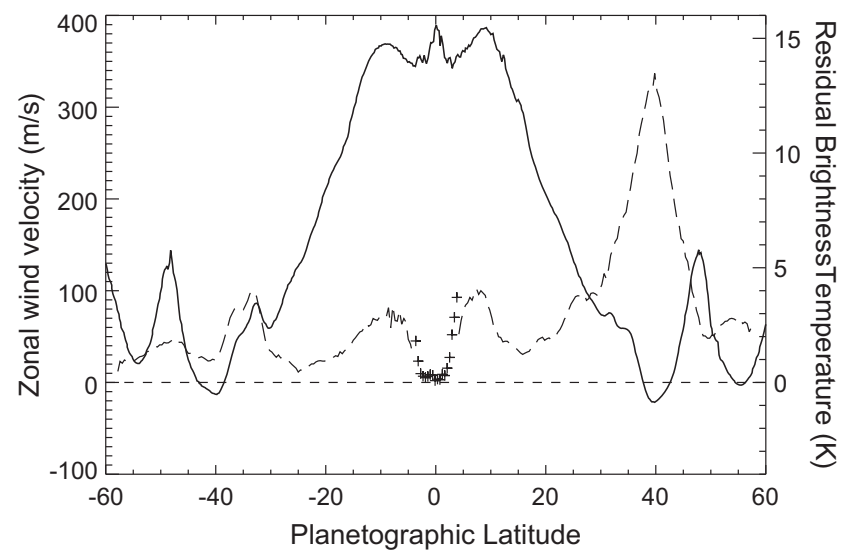

Fig. 14. Saturn zonal wind velocity (solid line) and mean residual temperature for the March 2011 map averaged over the storm longitudes (dashed line) as a function of planetographic latitude. From $-4^{\circ}$ to $+4^{\circ}$ the single December 2009 RPC scan is used (+ signs), as in Fig. 5. The zonal velocity profile used in this figure is from Cassini ISS data (García-Melendo et al., 2011). 
150-200 ppm at higher latitudes. Relative to our $3 \times$ solar reference state and not correcting for the removal of ammonia due to the $\mathrm{NH}_{4} \mathrm{SH}$ cloud, these correspond to $\mathrm{DF}_{5 \mathrm{bar}}=1.3$ at the equator, 0.31 at $\pm 8-10^{\circ}$, and $0.42-0.56$ at high latitudes. Our data require $\mathrm{DF}_{5 \mathrm{bar}}=1$ at the equator, $0.45-0.55$ at $\pm 8-10^{\circ}$ and $0.8-0.9$ at higher latitudes (also not correcting for the $\mathrm{NH}_{4} \mathrm{SH}$ cloud). Thus Fletcher et al. observe less ammonia in the subtropics and high latitudes and more ammonia at the equator than we do. They also see an increase in the ammonia abundance in the southern hemisphere between $-20^{\circ}$ and $-30^{\circ}$ which is consistent with the dip in brightness temperature that we observe there (Fig. 9). Given the differences in measurement techniques and altitudes covered, our results tend to agree with those of Fletcher et al. (2011a).

What dynamical mechanisms could cause a latitudinal thermal emission profile like the one we see in Fig. 9? We consider three possibilities. The first is that the brightness temperature variations are due to real latitudinal temperature variations, with the ammonia mixing ratio independent of latitude up to cloud base and saturated above. However, Fletcher et al. (2007) derived latitudinal temperature gradients on Saturn based on Cassini/CIRS observations, and the temperature contours near the equator are nearly flat, at least below the 0.5-bar level (their Fig. 2). In contrast, we observe brightness temperature fluctuations on the order of $10 \mathrm{~K}$ between the bands at $\pm 9^{\circ}$ and the equator. Fletcher et al. (2007) do observe a temperature dip in the equatorial region above the 0.4 bar level, with colder temperatures at the equator than in the subtropics by about $5 \mathrm{~K}$ in the $0.2-0.4$ bar region. This would be consistent with the brightness temperature pattern that we observe, but at altitudes above the 0.4-bar pressure level the density of ammonia gas is so small that we are not sensitive to these upper levels. The CIRS observations only provide temperatures above the 1 bar level so we cannot compare with the levels probed at $2.2 \mathrm{~cm}$.

In the cloud region, the ammonia concentration falls off according to the saturation vapor pressure (the Clausius-Clapeyron relation). When the atmospheric temperature decreases in this region, holding the $\mathrm{RH}$ constant at saturation, the ammonia concentration decreases and the weighting function moves to deeper (and warmer) levels, offsetting the decrease in temperature. Thus brightness temperature variations are buffered in the cloud layer, and we would not expect to see a large change in brightness temperature due to a change in atmospheric temperature. This is the same effect that we discussed in Section 2. To confirm this, we used our radiative transfer model to test the sensitivity of the $2.2-\mathrm{cm}$ brightness temperature to the model reference temperature at the 1-bar level. As we vary the reference temperature, the atmosphere remains saturated above the cloud base and the entire temperature profile shifts by approximately the same value as at the 1-bar level, including at the weighting function peak. In order to get brightness temperature variations on the order of $10 \mathrm{~K}$, the 1-bar temperature needs to be varied by $50 \mathrm{~K}$. The CIRS observations seem to rule out such large temperature swings from latitude to latitude, so we consider other options.

The second possibility is that upwelling in the equatorial region and downwelling on either side in each hemisphere produces an ammonia vapor distribution that is compatible with our observations. We postulate that air upwells at the equator, advecting ammonia-rich air from below, as suggested by previous authors to explain equatorial winds, composition, and clouds observed by Cassini (i.e. Yamakazi et al., 2005; Fletcher et al., 2011a). The ammonia precipitates out in the updrafts, and the dry air moves poleward, descending at latitudes out to $\pm 9^{\circ}$ in each hemisphere. This is the Hadley cell model. The rings of air moving poleward have to lose angular momentum to avoid spinning up while their distance to the rotation axis is decreasing. In other words, there has to be an eddy momentum flux (EMF) divergence within $\pm 9^{\circ}$ of the equator. On Earth, the region of EMF divergence extends to $\pm 30^{\circ}$, which is the poleward edge of the Hadley cell. The subtropics, which occupy the bands from $10^{\circ}$ to $30^{\circ}$ in each hemisphere, are marked by net downwelling and generally low relative humidity. They are "dry," like the bands near $\pm 9^{\circ}$ on Saturn. On Earth the tropospheric jet streams are located in the bands from $30^{\circ}$ to $40^{\circ}$ in each hemisphere, and the eddies, which arise from instability of the jet streams, are responsible for the EMF divergence at lower latitudes.

There are differences between the Hadley circulation on Earth and that on Saturn. First, in the published data there is only a hint of a zonal wind maximum, i.e., a jet stream, at $\pm 10^{\circ}$ on Saturn (García-Melendo et al., 2011), as shown in Fig. 14. At the equator there is a zonal wind maximum, which has no terrestrial analog. Poleward of $\pm 10^{\circ}$ there is EMF convergence, which pumps the equatorially superrotating jets on both Saturn and Jupiter (e.g. Ingersoll et al., 1981; Salyk et al., 2006; Del Genio et al., 2007). The Hadley cell model requires that the convergence become divergence within $\pm 9^{\circ}$ of the equator. This seems to work for Jupiter, which has zonal wind maxima at $\pm 6-7^{\circ}$ (planetocentric) and EMF divergence between $\pm 5^{\circ}$ (planetocentric), i.e., $\overline{u^{\prime} v^{\prime}}$ increasing with latitude from $-5^{\circ}$ to $+5^{\circ}$ (see Fig. 5 of Salyk et al., 2006). Whether it works for Saturn is uncertain, because trackable cloud features are scarce close to the equator and it has been impossible to measure the EMF there.

Lack of a solid surface to add angular momentum on the return flow is another difference between Earth and Saturn. On Earth, the low latitude easterlies gain westerly angular momentum from the surface as the air moves toward the equator. It is not clear that this would happen on a fluid planet. The return flow could be at any depth, and we do not know if rings of fluid exchange angular momentum there or not. Right now, the pieces of evidence for the Hadley cell model on Saturn are the high ammonia abundance at the equator, the low ammonia abundance and hint of zonal velocity maxima near $\pm 9^{\circ}$, and the observed EMF divergence in the band at Jupiter's equator.

Fletcher et al. (2011a) present evidence that two stacked meridional circulation cells, rotating in opposite directions, exist in Saturn's troposphere. From analysis of Cassini VIMS data, they find that deep $\mathrm{PH}_{3}$ and $\mathrm{AsH}_{3}$ show local maxima on either side of the equator, whereas the $\mathrm{PH}_{3}$ scale height, the upper cloud opacity, and the $\mathrm{NH}_{3}$ show local maxima at the equator. Stacked cells seem to explain the contradictory evidence of upwelling and downwelling both on and off the equator. As Fletcher et al. point out, the stacked cell hypothesis was originally invoked at Jupiter (Ingersoll et al., 2000; Gierasch et al., 2000; Showman and de Pater, 2005), where upper cloud opacity and $\mathrm{NH}_{3}$ indicated upwelling in the zones, but lightning and other evidence of moist convection indicated upwelling in the belts. Other authors also explore the possibility of meridionally overturning cells in Saturn's atmosphere (i.e. Del Genio et al., 2009). Since the present paper mainly concerns $\mathrm{NH}_{3}$, we do not attempt to synthesize all the Saturn data at this time.

\subsection{0-2011 Northern storm}

The third possibility is that there is some process in giant planet atmospheres that causes the "drying out" following convective events. The northern storm in the March 2011 map is very dry with respect to ammonia vapor (Fig. 13). What is it about the storm that produces such low ammonia $\mathrm{RH}$ ? The head of the storm is at $40^{\circ}$, $180^{\circ} \mathrm{W}$ (Sayanagi et al., 2013), and is indicated by the red triangle at the top of the figure in Fig. 13b. The green to blue trail to the west of the head is the tail of the storm, which wrapped all the way around the planet until it collided with the head in February 2011 (Fischer et al., 2011). By March 20, 2011, when this 2.2-cm data was collected, the storm had been in existence for almost 
4 months. The storm was a copious producer of lightning, which was detected at radio frequencies (Fischer et al., 2011) and in visible light (Dyudina et al., in preparation). Fig. 13 shows that, although the storm is very dry everywhere $(\mathrm{RH} \leqslant 0$, Fig. $13 \mathrm{a})$, there is also quite a bit of brightness temperature structure within the storm, which may be compatible with the Sánchez-Lavega et al. (2012) description of the three branches of the storm and the wave-like patterns within the storm tail.

Fig. 13b shows images taken by the Cassini imaging science subsystem (ISS) on March 7 and March 17, 2011 (Sayanagi et al., 2013), with the corresponding pieces of the RADAR maps below them (from March 20, 2011). The top panel of Fig. 13b spans $0-200^{\circ} \mathrm{W}$ longitude, and the bottom panel spans $60-130^{\circ} \mathrm{W}$ longitude. Both panels show $24.5-45^{\circ}$ planetographic latitude $\left(20.5-40^{\circ}\right.$ planetocentric). Red, green and blue color channels correspond to Cassini ISS camera's CB2 $(750 \mathrm{~nm})$, MT2 $(727 \mathrm{~nm})$, and MT3 $(889 \mathrm{~nm})$ filters, respectively, and they convey the cloud top heights. The altitude of the features generally increases in the order of red-green-blue (Sayanagi et al., 2013). There are no images closer in time to the March 20, 2011 radiometer image in Fig. 13a, for several reasons. Most important, the two instruments point along different axes of the spacecraft and cannot take data simultaneously. Also, the 2.2-cm map is taken near closest approach, when the field of view of the wide-angle camera is $15-20^{\circ}$ latitude, which is a small fraction of the planet. Finally, the observations were scheduled months in advance, long before the advent of the storm. Thus the detailed features in Fig. 13a are not necessarily the same features that appear in the ISS images in Fig. 13b. Some features, however, seem to be captured by the RADAR map, for example the anticyclonic vortex appears as a circular region of low $\mathrm{RH}$ (dark blue) near $15^{\circ} \mathrm{W}$. For an example of the changes that take place over an 11-h period, see Fischer et al. (2011).

Fig. 14 shows the zonal wind velocity (solid line) as a function of latitude with the March 2011 residual temperature averaged over the longitudes of the storm (dashed line) overlaid on it. The + signs between $-4^{\circ}$ and $4^{\circ}$ depict the December 2009 RPC scan brightness residuals used in Fig. 9. Storm alley in the southern hemisphere lies in the westward jet between $-40^{\circ}$ and $-45^{\circ}$. The northern storm is also located in a westward jet, but in the opposite hemisphere. The head of the storm is centered in the middle of the westward jet near $41^{\circ}$ ( $35^{\circ}$ planetocentric, Fischer et al., 2011). Some of the depletion of ammonia vapor by the northern storm gets pushed slightly to the north of the westward jet, which is consistent with work by Fletcher et al. (2011b) and Sánchez-Lavega et al. (2012), who observe two eastward branches to the north and south of the storm, with ammonia vapor depletion in the southern branch (Fletcher et al., 2011b). This pattern may be analogous to the storms in the southern hemisphere which are to the south of the westward jet (Porco et al., 2005). We suspect that the mechanism for "drying out" the small storms in the southern hemisphere and the 2010-2011 northern storm are the same. Ammonia depletion is consistent with the ISS (imaging science subsystem) team's interpretation of their observations of the southern storms at near-IR wavelengths. To measure clouds at various altitudes, they use three filters, like in Fig. 13b, where the absorption by methane gas is strong, weak, and negligible, respectively (Porco et al., 2005; Dyudina et al., 2007). From their observations they infer that on the first day the storms are active convective regions of optically thick, high clouds. On the third or fourth day they become stable circular clouds with optically thin, high haze above dark regions with no deep clouds - they become holes in the clouds. If the clouds are made of ammonia ice, then a hole in the clouds is consistent with depletion of ammonia vapor.

The VIMS (visible and infrared imaging spectrometer) team uses 352-bandpass spectra ranging from 0.35 to $5.1 \mu \mathrm{m}$, although the instrument has other ranges and resolutions. The dark regions seen in the near-IR are dark at all wavelengths, and Baines et al. (2009) interpret them as carbon-impregnated water frost rather than absence of deep clouds, the latter being the ISS interpretation. The carbon could come from dissociation of methane by lightning, since these are lightning clouds (Dyudina et al., 2007; Fischer et al., 2011).

Both the ISS description and the VIMS description seem to explain the dark color of the convective clouds after the third day. The low RH of ammonia inferred from the $2.2-\mathrm{cm}$ observations favors a hole in the clouds if the cloud particles are ammonia ice. The 2010-2011 northern storm, whose head resembles the high, thick convective clouds in ISS images, and whose tail is depleted in ammonia in the 2.2-cm maps, also supports the hole-in-the-cloud interpretation. Of course, the tail could be depleted in ammonia clouds and ammonia vapor but still have carbon-impregnated water ice clouds. The VIMS spectra of the head and tail of the northern storm will help resolve these different interpretations.

Convective storms seem to evolve into ammonia-poor regions, both in the southern hemisphere hot spots and in the northern storm. Also, the remnant of the northern storm is bright at $5 \mu \mathrm{m}$, which signals "an unusual dearth of deep clouds" (Baines, private communication, 2012). This agrees with the ISS interpretation of the southern hemisphere dark spots - that deep clouds are absent. So the question arises, why should convection "dry out" the atmosphere - removing gaseous ammonia and deep clouds? The answer may be in the nature of convection in hydrogen-helium atmospheres, in particular in the effect of mass loading by molecules of $\mathrm{NH}_{3}, \mathrm{H}_{2} \mathrm{~S}$, and $\mathrm{H}_{2} \mathrm{O}$, which have higher molecular mass than the ambient atmosphere. The parcels that have lost their load of the more massive molecules will have the most buoyancy, and they might rise the highest. These parcels would look "dry" when viewed from the top of the atmosphere. Mass loading might also explain the intermittency of convection on Saturn, but the details have yet to be worked out.

There are several models of moist convection in giant planet atmospheres. Some are axially symmetric (Yair et al., 1995a,b), and some are three dimensional (Hueso and Sánchez-Lavega, 2004), with the possibility of wind shear and precipitation on one side of the central updraft. These models start with an unstable initial state and follow the convective plume as it develops over a period of several hours or 1 day. Other models incorporate cumulus parameterizations into giant planet general circulation models (Del Genio and McGrattan, 1990; Palotai and Dowling, 2008). Sánchez-Lavega et al. (2012) studied a mass source in a shear flow patterned after the westward jet at $40^{\circ}$ planetographic that produces a GWS (Great White Spot) long tail. To the best of our knowledge, none of these models capture the 20-30 year cycle of planet-encircling storms or the ammonia depletion following convective events.

\section{Conclusions}

J13 used the 2.2-cm brightness temperature observations of Saturn presented in conjunction with radiative transfer calculations to produce residual brightness temperature maps for five dates between 2005 and 2011. In this work we analyzed the maps by making adjustments to the vertical ammonia distribution using the JAMRT program. We find that ammonia vapor must be depleted below the cloud base in some regions in order to obtain temperatures in agreement with observations. The observed brightness temperatures are consistent with a deep abundance of $3-4 \times$ solar ammonia (3.6-4.8 $\times 10^{-4}$ volume mixing ratio) with varying depletion factors relative to the standard model, which is saturated above cloud base. The depletion must extend to 2 bars or deeper for brightness temperatures $>160 \mathrm{~K}$. To obtain these 
results, we assume that Saturn's latitudinal temperature profile is constant in our sensitivity range of $0.5-2$ bars. The highest brightness temperatures we see are in the 2010-2011 northern storm $(165.7 \mathrm{~K})$ and in the subtropical latitudes of the October 2009 map ( $167 \mathrm{~K})$. The most striking feature, evident in Fig. 9, is the difference in the $2.2-\mathrm{cm}$ brightness temperature right at the equator versus that just off the equator. This implies that there are some interesting atmospheric dynamics at play in the equatorial region.

We presented three options to explain the brightness temperature pattern observed at $2.2-\mathrm{cm}$. One is that it represents real temperature variations, i.e., temperature variations from place to place at constant levels in the atmosphere. In principle, brightness temperature variations can be due to both physical temperature and absorber concentrations; however, the buffering effect makes the former option unlikely in our case. The second option is that large-scale upwelling and downwelling, like the Earth's Hadley circulation, creates dry zones like the subtropics of Earth. However this option does not explain the drying that follows the small-scale convective events in the southern hemisphere. The third option, which we do not explore in any depth, is that the drying is an intrinsic property of convection in giant planet atmospheres, and that it applies not just to the small southern lightning storms but also to the northern storm of 2010-2011. The subtropical dry bands are not copious producers of lightning and moist convection, so the third option might not apply there. It may that that the meridional circulation explanation applies to the subtropics, and the deep convection explanation applies to the lightning storms.

Modeling of atmospheric circulations on giant planets has proven difficult, and the lack of observational data in the deep atmosphere below the clouds is a limitation. It is important to try to reconcile the atmospheric motions with latitudinal distributions of tropospheric gases such as $\mathrm{NH}_{3}$ and $\mathrm{PH}_{3}$. This paper provides some insight into the distribution of ammonia vapor, with the hope that more work can be done with these observations to reconcile them with the energy and momentum balances of Saturn's atmosphere.

\section{Acknowledgments}

This research was conducted at the California Institute of Technology under contract with the National Aeronautics and Space Administration (NASA). It is partly based upon work supported by NASA under Grant No. 10-CDAP10-0051 issued through the Cassini Data Analysis and Participating Scientist (CDAPS) Program. A. Laraia was partially funded by a National Science Foundation (NSF) Graduate Research Fellowship Program (GRFP) fellowship. We acknowledge Sushil Atreya, Lena Adams, and Virgil Adumantroi for their invaluable contributions to the Juno atmospheric microwave radiative transfer (JAMRT) program. We would also like to acknowledge Kevin Baines for useful discussions on comparing RADAR and VIMS data.

\section{References}

Allison, M., 1990. Planetary waves in Jupiter's equatorial atmosphere. Icarus 83, 282-307.

Arregi, J., Rojas, J.F., Sánchez-Lavega, A., Morgado, A., 2006. Phase dispersion relation of the $5-\mu \mathrm{m}$ hot spot wave from a long-term study of Jupiter in the visible. J. Geophys. Res. 111 (E09010), 1-10.

Atreya, S.K., 2010. Atmospheric moons Galileo would have loved. In: Galileo's Medicean Moons - Their Impact on 400 years of Discovery. Cambridge University Press, pp. 130-140 (Chapter 16).

Baines, K.H. et al., 2009. Storm clouds on Saturn: Lightning-induced chemistry and associated materials consistent with Cassini/VIMS spectra. Planet. Space Sci. 57 1650-1658.

Briggs, F.H., Sackett, P.D., 1989. Radio observations of Saturn as a probe of its atmosphere and cloud structure. Icarus 80, 77-103.

de Pater, I., Massie, S.T., 1985. Models of the millimeter-centimeter spectra of the giant planets. Icarus 62, 143-171. de Pater, I., Dunn, D., Romani, P., Zahnle, K., 2001. Reconciling Galileo probe data and ground-based radio observations of ammonia on Jupiter. Icarus 149, 66 78.

Del Genio, A.D., McGrattan, K.B., 1990. Moist convection and the vertical structure and water abundance of Jupiter's atmosphere. Icarus 84, 29-53.

Del Genio, A.D., Barbara, J.M., Ferrier, J., Ingersoll, A.P., West, R.A., Vasavada, A.R., Spitale, J., Porco, C.C., 2007. Saturn eddy momentum fluxes and convection: First estimates from Cassini images. Icarus 189, 479-492.

Del Genio, A.D. et al., 2009. Saturn atmospheric structure and dynamics. In: Saturn from Cassini-Huygens. Springer, pp. 113-159 (Chapter 6).

Dyudina, U.A., Ingersoll, A.P., Ewald, S.P., Porco, C.C., Fischer, G., Kurth, W., Desch, M. Del Genio, A., Barbara, J., Ferrier, J., 2007. Lightning storms on Saturn observed by Cassini ISS and RPWS during 2004-2006. Icarus 190, 545-555.

Elachi, C. et al., 2004. RADAR: The Cassini Titan radar mapper. Space Sci. Rev. 115, $71-110$.

Fischer, G., Desch, M.D., Zarka, P., Kaiser, M.L., Gurnett, D.A., Kurth, W.S., Macher, W. Rucker, H.O., Lecacheux, A., Farrell, Cecconi, B., 2006. Saturn lightning recorded by Cassini/RPWS in 2004. Icarus 183, 135-152.

Fischer, G., Kurth, W.S., Dyudina, U.A., Kaiser, M.L., Zarka, P., Lecacheux, A., Ingersoll, A.P., Gurnett, D.A., 2007. Analysis of a giant lightning storm on Saturn. Icarus $190,528-544$.

Fischer, G. et al., 2011. A giant thunderstorm on Saturn. Nature 475, 75-77.

Fletcher, L.N., Irwin, P.G.J., Teanby, N.A., Orton, G.S., Parrish, P.D., de Kok, R., Howett, C., Calcutt, S.B., Bowles, N., Taylor, F.W., 2007. Characterising Saturn's vertical temperature structure using Cassini/CIRS. Icarus 189, 457-478.

Fletcher, L.N., Baines, K.H., Momary, T.W., Showman, A.P., Irwin, P.G.J., Orton, G.S. Roos- Serote, M., Merlet, C., 2011a. Saturn's tropospheric composition and clouds from Cassini/VIMS 4.6-5.1 $\mu \mathrm{m}$ nightside spectroscopy. Icarus 214, 510533.

Fletcher, L.N. et al., 2011b. Thermal structure and dynamics of Saturn's northern springtime disturbance. Science 332, 1413-1417. http://dx.doi.org/10.1126/ science. 1204774

Fletcher, L.N. et al., 2012. Sub-millimetre spectroscopy of Saturn's trace gases from Herschel/SPIRE. Astron. Astrophys. 539, A44. http://dx.doi.org/10.1051/0004$6361 / 201118415$.

García-Melendo, E., Pérez-Hoyos, S., Sánchez-Lavega, A., Hueso, R., 2011. Saturn's zonal wind profile in 2004-2009 from Cassini ISS images and its long-term variability. Icarus 215, 62-74.

Gierasch, P.J. et al., 2000. Observation of moist convection in Jupiter's atmosphere. Nature 403, 628-630.

Grevesse, N., Asplund, M., Sauval, A.J., 2005. The new solar chemical composition. EAS Publ. Ser. 17, 21-30. http://dx.doi.org/10.1051/eas:2005095.

Grossman, A.W., Muhleman, D.O., Berge, G.L., 1989. High resolution microwave observations of Saturn. Science 245, 1211-1215.

Hueso, R., Sánchez-Lavega, A., 2004. A three-dimensional model of moist convection for the giant planets. II: Saturn's water and ammonia moist convective storms. Icarus 172, 255-271.

Ingersoll, A., Beebe, R., Mitchell, J., Garneau, G., Yagi, G., Muller, J., 1981. Interaction of eddies and mean zonal flow on Jupiter as inferred from Voyager 1 and 2 images. J. Geophys. Res. 86, 8733-8743.

Ingersoll, A.P., Gierasch, P.J., Banfield, D., Vasavada, A.R.Galileo Imaging Team, 2000 Moist convection as an energy source for the large-scale motions in Jupiter's atmosphere. Nature 403, 630-632.

Janssen, M.A., Hofstadter, M.D., Gulkis, S., Ingersoll, A.P., Allison, M.D., Bolton, S.J., Kamp, L.W., 2005. Microwave remote sensing of Jupiter's atmosphere from an orbiting spacecraft. Icarus $173,447-453$.

Janssen, M.A., Lorenz, R.D., West, R., Paganelli, F., Lopes, R.M., Kirk, R.L., Elachi, C. Wall, S.D., Johnson, W.T.K., Anderson, Y., Boehmer, R.A., Callahan, P., Gim, Y., Hamilton, G.A., Kelleher, K.D., Roth, L., Stiles, B., Le Gall, A.the Cassini Radar Team, 2009. Titan's surface at 2.2-cm wavelength imaged by the Cassini RADAR radiometer: Calibration and first results. Icarus 200, 222-239.

Janssen, M.A., Ingersoll, A.P., Allison, M.D., Gulkis, S., Laraia, A.L., Baines, K. Edgington, S., Anderson, Y., Kelleher, K., Oyafuso, F., 2013. Saturn's thermal emission at 2.2-cm wavelength as imaged by the Cassini RADAR radiometer Icarus, this issue.

Kaiser, M.L., Connerney, J.E.P., Desch, M.D., 1983. Atmospheric storm explanation of saturnian electrostatic discharges. Nature 303, 50-53.

Lindal, G.F., Sweetnam, D.N., Eshleman, V.R., 1985. The atmosphere of Saturn: An analysis of the Voyager radio occultation measurements. Astron. J. 90, 11361146.

Palotai, C., Dowling, T.E., 2008. Addition of water and ammonia cloud microphysics to the EPIC model. Icarus 194, 303-326.

Porco, C.C. et al., 2005. Cassini imaging science: Initial results on Saturn's atmosphere. Science 307, 1243-1247.

Salyk, C., Ingersoll, A.P., Lorre, J., Vasavada, A., Del Genio, A.D., 2006. Interaction between eddies and mean flow in Jupiter's atmosphere: Analysis of Cassin imaging data. Icarus $185,430-442$.

Sánchez-Lavega, A., Rojas, J.F., Sada, P.V., 2000. Saturn's zonal winds at cloud level Icarus $147,405-420$

Sánchez-Lavega, A. et al., 2011. Deep winds beneath Saturn's upper clouds from a seasonal long-lived planetary-scale storm. Nature 475, 71-74.

Sánchez-Lavega, A., del Río-Gaztelurrutia, T., Delcroix, M., Legarreta, J.J., GómezForrellad, J.M., Hueso, R., García-Melendo, E., Pérez-Hoyos, S., BarradoNavascués, D., Lillo, J.International Outer Planet Watch Team IOPW-PVOL 2012. Ground-based observations of the long-term evolution and death of Saturn's 2010 Great White Spot. Icarus 220, 561-576. 
Sayanagi, K., Dyudina, U.A., Ewald, S.P., Fischer, G., Ingersoll, A.P., Kurth, W.S., Muro, G.D., Porco, C.C., West, R.A., 2013. Dynamics of Saturn's great storm of 20102011 from Cassini ISS and RPWS. Icarus 223, 460-478.

Showman, A.P., de Pater, I., 2005. Dynamical implications of Jupiter's tropospheric ammonia abundance. Icarus 174, 192-204.

West, R.A., Baines, K.H., Karkoschka, E., Sánchez-Lavega, A., 2009. Clouds and aerosols in Saturn's atmosphere. In: Saturn from Cassini-Huygens. Springer, pp. 161-179 (Chapter 7).
Yair, Y., Levin, Z., Tzivion, S., 1995a. Microphysical processes and dynamics of a jovian thundercloud. Icarus 114, 278-299.

Yair, Y., Levin, Z., Tzivion, S., 1995b. Lightning generation in a jovian thundercloud: Results from an axisymmetric numerical cloud model. Icarus 115, 421-434.

Yamakazi, Y.H., Read, P.L., Skeet, D.R., 2005. Hadley circulations and Kelvin wavedriven equatorial jets in the atmospheres of Jupiter and Saturn. Planet. Space Sci. 53, 508-525. 